\title{
Scaled solar tracks and isochrones in a large region of the $Z-Y$ plane
}

\author{
II. From 2.5 to $20 M_{\odot}$ stars $^{\star}$ \\ G. Bertelli ${ }^{1}$, E. Nasi ${ }^{1}$, L. Girardi ${ }^{1}$, and P. Marigo ${ }^{2}$ \\ 1 INAF - Padova Astronomical Observatory, Vicolo dell'Osservatorio 5, 35122 Padova, Italy \\ e-mail: gianpaolo.bertelli@oapd.inaf.it \\ 2 Astronomy Department, Padova University, Vicolo dell'Osservatorio 3, 35122 Padova, Italy
}

Received 17 March 2009 / Accepted 17 September 2009

\begin{abstract}
We extend our theoretical computations for low-mass stars to intermediate-mass and massive stars, for which few databases exist in the literature. Evolutionary tracks and isochrones are computed for initial masses $2.50-20 M_{\odot}$ for a grid of 37 chemical compositions with metal content $Z$ between 0.0001 and 0.070 and helium content $Y$ between 0.23 and 0.40 to enable users to obtain isochrones for ages as young as about $10^{7}$ years and to simulate stellar populations with different helium-to-metal enrichment laws. The Padova stellar evolution code is identical to that used in the first paper of this series. Synthetic TP-AGB models allow stellar tracks and isochrones to be extended until the end of the thermal pulses along the AGB. We provide software tools for the bidimensional interpolation (in $Y$ and $Z$ ) of the isochrones from very old ages down to about $10^{7}$ years. This lower limit depends on chemical composition. The extension of the blue loops and the instability strip of Cepheid stars are compared and the Cepheid mass-discrepancy is discussed. The location of red supergiants in the H-R diagram is in good agreement with the evolutionary tracks for masses from 10 to $20 M_{\odot}$. Tracks and isochrones are available in tabular form for the adopted grid of chemical compositions in the extended plane $Z-Y$ in three photometric systems. An interactive web interface allows users to obtain isochrones of any chemical composition inside the provided $Z-Y$ range and also to simulate stellar populations with different $Y(Z)$ helium-to-metal enrichment laws.
\end{abstract}

Key words. stars: evolution - Cepheids

\section{Introduction}

In general, the helium content of stellar populations is poorly known. For super-solar metallicities, the helium content is unconstrained, and has usually been derived from simple reasoning, based on naive chemical evolution models of galaxies, rather than observational grounds.

The most popular assumption in evolutionary models of stars is that $Y$ increases linearly with $Z$. This assumption is inaccurate for old globular clusters, some of which contain multiple stellar populations with evidence of significant helium enrichment (Piotto et al. 2007; Piotto 2009). Even the solar initial content of helium has been debated, since the values derived from helioseismology depend on the assumed efficiency of helium diffusion (Guzik et al. 2005, 2006; Basu \& Antia 2008), whereas on the other hand helioseismology has been challenged by the debate about the oxygen abundance (Montalban et al. 2004; Scott et al. 2009). For these reasons it is likely that the helium content of stellar evolutionary models will in the future be revised many times. An alternative approach, followed in this paper, is that of computing stellar models for a significant range of the $Z-Y$ plane, so that interpolations in the grid allow us to consider many possible $Y(Z)$ relations.

* Isochrone tables are only available in electronic form at the CDS via anonymous ftp to cdsarc.u-strasbg.fr $(130.79 .128 .5)$ or via http://cdsweb.u-strasbg.fr/cgi-bin/qcat?J/A+A/508/355
A large amount of theoretical investigation has been devoted to the evolution of low-mass stars populating old galactic globular clusters, since rich stellar clusters provide us with the opportunity to test the results of stellar evolution theories. The increasing amount of data for young populous clusters in both of the Magellanic Clouds encourages us to pay special attention to the evolutionary behaviour of more massive stars presently evolving in those clusters. However, not so much theoretical computation has been addressed to the implementation of existing stellar model databases for more massive stars, as done for low masses.

The Padova and Geneva groups originally compiled grids of stellar models with overshooting up to $120 M_{\odot}$ for several chemical compositions (Bressan et al. 1993; Fagotto et al. 1994a,b; and relative isochrones in Bertelli et al. 1994; Schaller et al. 1992; Schaerer et al. 1993a,b; Charbonnel et al. 1993; Meynet et al. 1994). Girardi et al. (2000) computed a new set of low- and intermediate-mass stellar models with updated opacities and equation of state. Bono et al. (2000) presented intermediate-mass standard models with different helium and metal content ( $3 \leq M \leq 15 M_{\odot}$ ), and Pietrinferni et al. (2004, 2006) database makes available stellar models and isochrones for scaled-solar and $\alpha$-enhanced metal distributions in the mass range between 0.5 and $10 M_{\odot}$ with and without overshooting.

Following the first release of the new stellar evolution models of the Padova database for low-mass stars in a large region of the $Z-Y$ plane by Bertelli et al. (2008, hereinafter Paper I), in 
this paper we present models for stars from 2.5 to $20 M_{\odot}$ with the same range of chemical compositions as in Paper I. Among the astrophysical problems of interest in this range of mass, we discuss whether observations of Cepheids in the Magellanic Clouds (MCs) and the Galaxy can be reproduced by these new models. Massive red supergiants in the Galaxy and in MCs with newly derived physical parameters by Levesque et al. $(2005,2006)$ are compared with our models from 10 to $20 M_{\odot}$, evaluating the adequacy of stellar models in this mass range.

A neglected process in our massive stellar models is stellar rotation. Significant advances have been made in this field (e.g., Heger \& Langer 2000; Meynet \& Maeder 2000), which demonstrated that the evolutionary paths of rapidly rotating massive stars can differ from those of non-rotating stars. A book by Maeder (2009) on the physics, formation, and evolution of rotating stars presents a very detailed description of the effects of rotation on stellar evolution. Rapid rotation can trigger strong mixing inside massive stars, extend the core hydrogen-burning lifetime, significantly increase the luminosity, and change the chemical composition at the stellar surface with time. Regardless of the success of rotating stellar models in explaining a variety of stellar phenomena, rotational mixing is still a matter of debate (de Mink et al. 2009).

Our choice of neglecting stellar rotation in this set of models has two main motivations, namely:

1. not all stars begin their life with the same rotational velocity;

2. stars have rotational axes that are not all oriented in the same direction.

These two stochastic effects introduce some dispersion in the distribution of the stars in the HR disgram, in contradiction with the deterministic character of stellar isochrones. The "nonrotating isochrones" represent the starting point for any further study.

The present paper is organized as follows. In Sect. 2, we summarize the main input physics and in Sect. 3, TP-AGB models are described. In Sect. 4, stellar tracks are presented with a description of tables indicating changes in surface chemical composition after the first and the second dredge-up. Isochrones and relative tables are described in Sect. 5, in addition to the interpolation scheme allowing to obtain isochrones and to simulate stellar populations in a large region of the $Z-Y$ plane. Cepheids and the problem of the mass discrepancy are discussed in Sect. 6. Section 7 deals with massive red supergiants in the Galaxy and the Magellanic Clouds, and Sect. 8 presents the concluding remarks.

\section{Input physics and coverage of the $Z-Y$ plane}

The stellar evolution code adopted in this work is the same as in Bertelli et al. (2008, hereinafter Paper I ). The masses described in this paper are between 2.5 and $20 M_{\odot}$, to ensure that we have a complete stellar model database from 0.15 to $20 M_{\odot}$. In the following, we summarize the input physics described in Paper I.

\subsection{Initial masses and chemical compositions}

The first release of the new evolutionary models was presented in Paper I for masses from 0.15 to $2.5 M_{\odot}$ (Bertelli et al. 2008). The stellar models were computed for initial masses 2.5, 3, 3.5, 4, 4.5, 5, 6, 7, 8, 10, 12, 15, and $20 M_{\odot}$, and, as in Paper I, from the ZAMS to the end of helium burning. The initial chemical composition is in the range $0.0001 \leq Z \leq 0.070$
Table 1. Combinations of $Z$ and $Y$ of the computed tracks.

\begin{tabular}{cccccc}
\hline \hline$Z$ & $Y 1$ & $Y 2$ & $Y 3$ & $Y 4$ & $Y 5$ \\
\hline 0.0001 & 0.23 & 0.26 & 0.30 & & 0.40 \\
0.0004 & 0.23 & 0.26 & 0.30 & & 0.40 \\
0.001 & 0.23 & 0.26 & 0.30 & & 0.40 \\
0.002 & 0.23 & 0.26 & 0.30 & & 0.40 \\
0.004 & 0.23 & 0.26 & 0.30 & & 0.40 \\
0.008 & 0.23 & 0.26 & 0.30 & 0.34 & 0.40 \\
0.017 & 0.23 & 0.26 & 0.30 & 0.34 & 0.40 \\
0.040 & & 0.26 & 0.30 & 0.34 & 0.40 \\
0.070 & & & 0.30 & 0.34 & 0.40 \\
\hline
\end{tabular}

for the metal content, and for the helium content in the range $0.23 \leq Y \leq 0.40$ as shown in Table 1 . In addition, tracks were computed for masses between 4.5 and $20 M_{\odot}$ with $Z=0.030$ and $Y=0.26,0.30,0.34$, and 0.40 to derive more reliable interpolated isochrones for more massive stars.

\subsection{The choice of scaled-solar abundances}

For each value of $Z$, the fractions of different metals follow a scaled solar distribution, as compiled by Grevesse \& Noels (1993), and adopted in the OPAL opacity tables. The ratio of abundances of different isotopes is assumed to be identical to Anders \& Grevesse (1989).

In this regard, we note that both the solar composition, and what is referred to as the alpha-enhanced distribution of metals have changed, by factors of up to $0.3 \mathrm{dex}$, in the past few years. Moreover, there is also an increasing perception that neither scaled solar, nor alpha-enhanced compositions are universal: they apply only to particular regions of spiral galaxies that are similar to our own. For instance, there are documented cases of slightly alpha-depleted composition in some areas of the LMC (e.g., Pompéia et al. 2008). For nearby dwarf galaxies, the modern observational picture is that their populations are in general slightly alpha-depleted for $[\mathrm{Fe} / \mathrm{H}]>-1.0$, becoming alphaenhanced only at very low metallicities (see Fig. 11 in Tolstoy et al. 2009, and references therein). On the high-metallicity end, although it is generally believed that the most massive ellipticals are metal-rich and alpha-enhanced, there is no guarantee that they follow the same distribution of alpha-elements as nearby halo stars.

The above-mentioned observations of dwarf galaxies demonstrate that low-metallicity models with scaled solar compositions are useful and, in many cases, appropriate, to the study of nearby galaxies. Since the observational view of a "typical alpha-enhanced composition" continues to change, we prefer to be conservative and begin the modelling from the most well studied case, the scaled solar one, which is reasonably wellknown, apart from the solar oxygen abundance. We will explore a few possible cases of alpha-enhancement in future papers. This approach is consistent with our choice of scaled solar abundances and the rules introduced by Salaris et al. (1993) to approximate low- $Z$ alpha-enhanced isochrones using scaled solar ones (which can be applied to the Milky Way halo, metal-poor globular clusters, and the metal-poorest stars in dwarf galaxies).

As far as the use of "old solar abundances" is concerned (Grevesse \& Noels 1993), we note the following:

- Some more recent studies (e.g., Caffau et al. 2008, and references therein) confirm the high $\mathrm{O}$ abundances typical of older compilations of solar abundances, rather than the low 
values claimed by Asplund et al. (2004). Solar models computed with low heavy-element abundances also strongly disagree with the constraints from helioseismology (see Basu \& Antia 2008, and references therein).

- We computed a few sequences of stellar tracks using several solar compositions. The difference between the models computed at the same mass, metallicity, and mixing length parameter was found to be negligible (as discussed in Sect. 4.3.1).

\subsection{Opacities}

The radiative opacities for scaled solar mixtures are assumed to be those of the OPAL group (Iglesias \& Rogers 1996) for temperatures higher than $\log T=4$, and the molecular opacities are those of Alexander \& Ferguson (1994) for $\log T<4.0$ as in Salasnich et al. (2000). For very high temperatures $(\log T \geq 8.7)$, we use the opacities of Weiss et al. (1990).

The conductive opacities of electron-degenerate matter are taken from Itoh et al. (1983). For the interpolation within the opacity tables grids in both papers, we used the two-dimensional bi-rational cubic damped-spline algorithm (see Schlattl \& Weiss 1998; Salasnich et al. 2000; and Weiss \& Schlattl 2000). In general, evolutionary models of low-mass stars differ very little by changing the opacity interpolation scheme. The model predictions for massive stars $\left(M \geq 5 M_{\odot}\right)$ are far more sensitive to the interpolation algorithm as discussed in Salasnich et al. (2000)

\subsection{Equation of state}

The equation of state (EOS) for temperatures higher than $10^{7} \mathrm{~K}$ is that of a fully-ionized gas, including electron degeneracy in the way described by Kippenhahn et al. (1967). The effect of Coulomb interactions between the gas particles at high densities is taken into account as described in Girardi et al. (1996). For temperatures lower than $10^{7} \mathrm{~K}$, the detailed "MHD" EOS of Mihalas et al. (1990, and references therein) is adopted. We note that the MHD EOS is critical only for stellar models of mass lower than $0.7 M_{\odot}$ during their main sequence evolution.

\subsection{Reaction rates and neutrino losses}

The reaction rates are taken from the compilation of Caughlan \& Fowler (1988, hereinafter CF88), apart from ${ }^{17} \mathrm{O}(\mathrm{p}, \alpha){ }^{14} \mathrm{~N}$ and ${ }^{17} \mathrm{O}(\mathrm{p}, \gamma){ }^{18} \mathrm{~F}$, for which we use the determinations by Landré et al. (1990). The uncertain ${ }^{12} \mathrm{C}(\alpha, \gamma){ }^{16} \mathrm{O}$ rate was set to be 1.7 times the values given by Caughlan \& Fowler (1988), as indicated by the study of Weaver \& Woosley (1993) of the nucleosynthesis by massive stars. The electron screening factors for all reactions are those from Graboske et al. (1973). The abundances of the various elements are evaluated with the aid of a semi-implicit extrapolation scheme, as described in Marigo et al. (2001). The energy losses by neutrinos are from Haft et al. (1994).

In Sect. 4.3.2 we discuss the effects on stellar models of measurements of the ${ }^{14} \mathrm{~N}(\mathrm{p}, \gamma){ }^{15} \mathrm{O}$ reaction rate by the LUNA experiment (Formicola et al. 2004).

\subsection{Convection}

The most important physical process for mixing in stars is convection, but at present convection remains a main source of uncertainty in stellar models computations. In our stellar models, convection is treated with the Schwarzschild criterion for stability. Usually energy transport in the outer convection zone of stars is described according to the mixing-length theory (MLT) of Böhm-Vitense (1958). We adopt the same value as in Paper I for the MLT parameter $(\alpha=1.68)$, calibrated by means of the solar model.

\subsubsection{Overshoot}

The extension of the convective regions in stellar models takes into account overshooting from the borders of both core and envelope convective zones (Bressan et al. 1981; Alongi et al. 1991; Girardi et al. 2000). In the following, we adopt the formulation by Bressan et al. (1981) in which the boundary of the convective core is defined to be the layer where the velocity (rather than the acceleration) of convective elements vanishes. This non-local treatment of convection requires the use of a free parameter related to the mean free path $l$ of convective elements defined to be $l=\Lambda_{\mathrm{c}} H_{\mathrm{p}},\left(H_{\mathrm{p}}\right.$ being the pressure scaleheight). The choice of this parameter determines the extent of the overshooting across the border of the classical core (determined by the Schwarzschild criterion). Other authors define the extent of the overshooting zone to be at the distance $d=\Lambda_{\mathrm{c}} H_{\mathrm{p}}$ above the border of the convective core (Schwarzschild criterion). The value 0.5 used in the Padova models is almost equivalent to the 0.2 value adopted by other groups (Meynet et al. 1994; Pietrinferni et al. 2004; Yi et al. 2001). The non-equivalence of the parameter used to describe the extension of convective overshooting by different groups has been a recurrent source of misunderstanding in the literature.

We adopt the same prescription as in Paper I (Bertelli et al. 2008) and in Girardi et al. (2000) for the parameter $\Lambda_{c}$ for $H-$ and He-burning stages. Overshooting at the lower boundary of convective envelopes is also considered. For $M>2.5 M_{\odot}$, a value of $\Lambda_{e}=0.7$ was assumed, as in Bertelli et al. (1994) and Girardi et al. (2000).

\subsubsection{Hydrogen semiconvection}

During the core H-burning phase of massive stars, radiation pressure and electron scattering opacity produce a large convective core surrounded by an H-rich region, which is potentially unstable to convection if the original gradient in chemical abundance remains, but stable if suitable mixing is allowed to occur. This region of the star is assumed in theoretical models to undergo sufficient mixing until the condition of neutrality is restored, to transport negligible energy flux. The Schwarzschild condition produces smoother chemical profiles and, in some cases, a fully intermediate convective layer. Similar instability also occurs during the early shell H-burning phase. Taking into account mass loss from massive stars, the extension of semiconvection and/or intermediate full convection at the top of the H-burning shell is much smaller than in constant-mass stellar models. The effects of H-semiconvection on the evolution of massive stars were summarized by Chiosi \& Maeder (1986).

\subsubsection{Helium semiconvection}

As He-burning proceeds in the convective core of low-mass stars during the early stages of the horizontal branch (HB), the size of the convective core increases. Once the central value of helium falls below $Y_{\mathrm{c}}=0.7$, the temperature gradient reaches a local minimum, so that continued overshoot is no longer able to 
restore the neutrality condition at the border of the core. The core divides into an inner convective core and an outer convective shell. As further helium is captured by the convective shell, the outer shell tends to become stable, leaving behind a region of varying composition in conditions of neutrality. This zone is called semiconvective. Starting from the centre and going outwards, the matter in the radiatively stable region above the formal convective core is mixed layer by layer until neutrality is achieved. This picture holds during most of the central He-burning phase. The extension of the semiconvective region varies with stellar mass, being important in low- and intermediate-mass stars up to about $5 M_{\odot}$, and negligible in more massive stars. We followed the scheme of Castellani et al. (1985), as described in Bressan et al. (1993).

\subsection{Mass loss}

Mass loss has a crucial impact on the evolution of massive stars, affecting evolutionary tracks, lifetimes, and surface abundances. The role of radiation pressure in driving mass loss from massive stars has been firmly accepted by theoretical astrophysics since the pioneering studies by Lucy \& Solomon (1970), and Castor et al. (1975). A relevant review on the evolution of massive stars with mass loss was given by Chiosi \& Maeder (1986), while Kudritzki \& Puls (2000) exhaustively describe winds from hot stars. The metallicity dependence of mass loss rates is usually included using the formula:

$$
\dot{M}(Z)=\dot{M}\left(Z_{\odot}\right)\left(Z / Z_{\odot}\right)^{\alpha}
$$

where the exponent $\alpha$ varies between values of $0.5-0.6$ (Kudritzki \& Puls 2000; Kudritzki 2002), and 0.7-0.86 (Vink et al. 2001; Vink \& de Koter 2005) for O-type and WR stars. A comparison between mass loss prescriptions and observed mass loss rates, and the observational dependence of the rate on the metal content of massive stars atmospheres was presented by Mokiem et al. (2007). They found a metallicity dependence consistent with previous values and stated that those $\dot{M}(Z)$ relations hold for stars more luminous than $\sim 10^{5.2} L_{\odot}$.

In this paper, we adopt the mass loss rates from stellar winds driven by radiation pressure according to de Jager et al. (1988) for all evolutionary stages starting from the main sequence and from about $\sim 6-7$ to $20 M_{\odot}$ (the upper mass limit to this evolutionary track release). The rates incorporate the dependence on metallicity given by Kudritzki et al. (1989)

$\dot{M}_{Z}=\left(Z / Z_{\odot}\right)^{0.5} \dot{M}_{Z_{\odot}}$.

In a future paper dealing with the evolution of massive stars, for the mass-loss rates we will adopt relationships based on more recent observations and/or determinations.

\section{Synthetic TP-AGB models}

An important update of the database of evolutionary tracks is the extension of stellar models and isochrones until the end of the thermal pulses along the asymptotic giant branch (TP-AGB) for all masses up to $\sim 6 M_{\odot}$. The evolution from the first thermal pulse to the complete ejection of the stellar envelope is computed with the synthetic code described in Marigo \& Girardi (2007, and references therein), to whom we refer for all details. It is important to mention that these TP-AGB tracks have been updated in many aspects, describing for instance the transition to the C-star phase by means of the third dredge-up event,

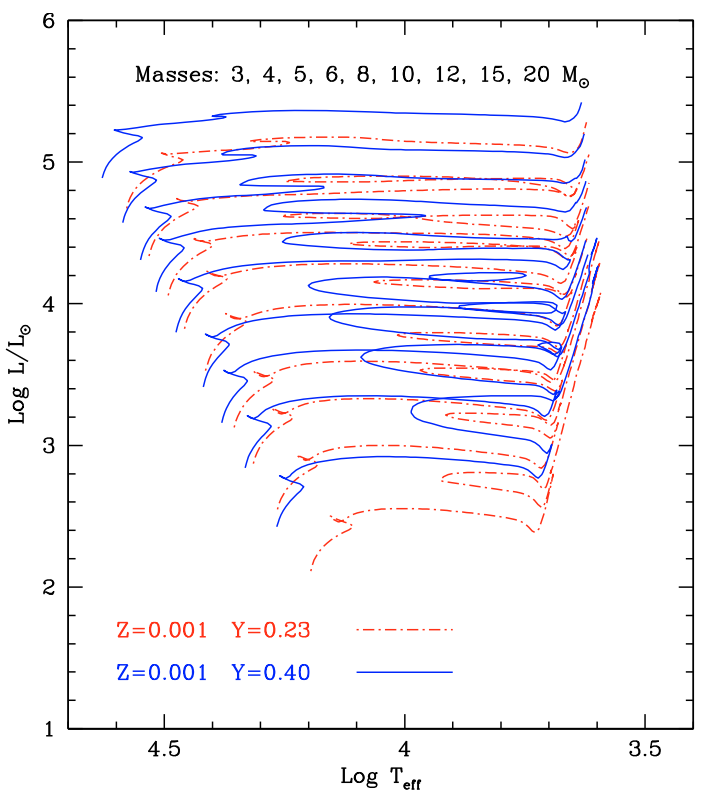

Fig. 1. Evolutionary tracks in the HR diagram for the composition $Z=$ $0.001, Y=0.23$ (dot-dashed line) and $Z=0.001, Y=0.40$ (solid line).

the prevention of this transition for the most massive tracks because of hot bottom burning, the proper effective temperatures of carbon stars, and suitable mass-loss rates of the $M$ and C-type stars. Although the models are not calibrated in the same way as Marigo \& Girardi (2007) tracks (i.e., present models might not reproduce the luminosity functions and lifetimes of carbon stars in the Magellanic Clouds), they represent a significant improvement to the simple way the TP-AGB tracks were included in many previous sets of isochrones.

\section{Stellar tracks}

\subsection{Evolutionary stages and mass ranges}

We consider that stellar models are on the zero age mainsequence (ZAMS) when chemically homogeneous stars begin their H-burning and the gravitational energy production is less than about $1 \%$ of the total energy. Our models are evolved from the ZAMS, and the evolution is followed in detail through the entire $\mathrm{H}$ - and He-burning phases. The tracks are stopped at the beginning of the TP-AGB phase (bTP-AGB) in intermediatemass stars, or at the carbon ignition in our more massive models. Stellar models and isochrones are extended until the end of the thermal pulses along the AGB with the synthetic TP-AGB models by Marigo \& Girardi (2007).

\subsection{Tracks in the HR diagram}

We display only some examples of the sets of computed evolutionary tracks (i.e., $Z=0.001$ for $Y=0.23$ and $Y=0.40$, and $Z=0.040$ for $Y=0.26$ and $Y=0.40$ ) for masses between 2.5 and $20 M_{\odot}$ from the ZAMS to the beginning of either the TP-AGB, or carbon burning. Figures 1 and 2 illustrate the range of temperatures and luminosities involved for the boundary values of helium content at a given metallicity in the theoretical HR diagram. These figures also illustrate the dependences of the extension of blue loops on both metallicity and helium content, which are significant at low metallicity, and practically absent for higher metal content $(Z \geq 0.040)$. The extension of 


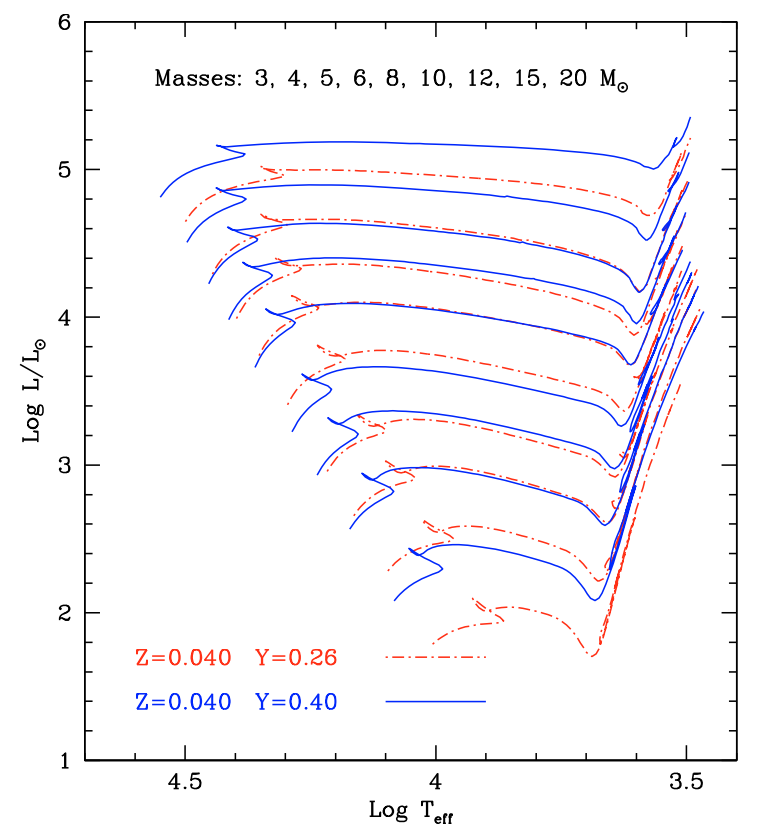

Fig. 2. Evolutionary tracks in the HR diagram for the composition $Z=$ $0.040, Y=0.26$ (dot-dashed line) and $Z=0.040, Y=0.40$ (solid line).

blue loops requires a detailed discussion since there are observations that are not easily reproduced by stellar models, such as Cepheids, and this topic is dealt with in Sect. 6. The location of red supergiants (RSG) on the H-R diagram did not always agree with the predictions of stellar evolutionary models, previousy appearing to be too cool and too luminous to coincide with the position of the evolutionary tracks. New effective temperatures and luminosities for the RSG populations of galaxies by Levesque et al. $(2005,2006)$ greatly improved the agreement between the RSGs and the evolutionary models, as is evident in Sect. 7 from the comparison with our new moderate massive models. The data tables for the present evolutionary tracks are available only in electronic format ${ }^{1}$.

\subsection{Testing the effects of different input physics}

We computed a few sequences for different initial chemical compositions, opacities, and for nuclear reaction rates from the LUNA experiment, obtaining very modest changes in the tracks, as discussed in the following.

\subsubsection{Initial chemical composition and opacities}

Updating both the chemical mixture (EOS) and the lowtemperature opacities (determined with the AESOPUS code by Marigo \& Aringer 2009), we computed a few sequences of stellar tracks using the distribution of element abundances of the solar chemical composition according to a) Grevesse \& Noels (1993), b) Grevesse \& Sauval (1998), and c) Grevesse et al. (2007). The differences between the models for the same $Z$ are negligible. To complete additional comparison, we also used the opacity tables by Alexander \& Ferguson (1994), with reference to the Grevesse (1991) solar composition. We found that the differences between the models, computed at the same mass,

\footnotetext{
${ }^{1}$ A website with the complete data-base (including additional data and future extensions) will be maintained at http://stev.oapd.inaf. it/YZVAR with suitable information on electronic tables (the same as in Paper I).
}

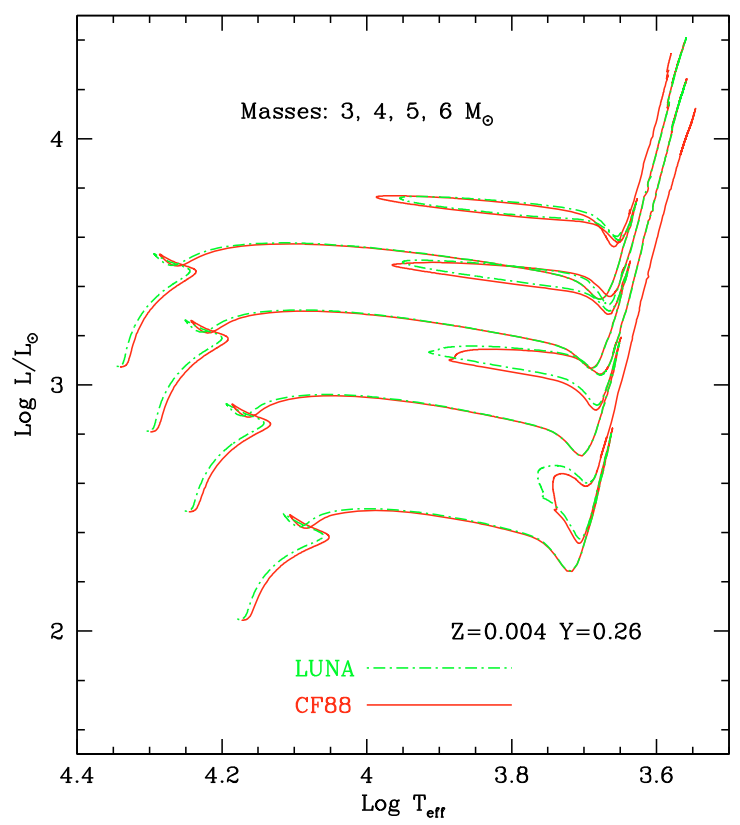

Fig. 3. Evolutionary tracks in the HR diagram for the composition $Z=0.004, Y=0.26$ computed with the new LUNA rate for the ${ }^{14} \mathrm{~N}(\mathrm{p}, \gamma){ }^{15} \mathrm{O}$ nuclear reaction (dot-dashed line) and with the rate according to Caughlan \& Fowler (1988) (solid line).

metallicity and mixing-length parameter, are completely negligible. In particular, the corresponding giant branches in the H-R diagram practically overlap, the typical separation at fixed luminosity being of the order of 0.002 dex or less throughout their extension.

\subsubsection{Nuclear reaction rates}

In our models, we tested the effect of measurements of the ${ }^{14} \mathrm{~N}(\mathrm{p}, \gamma){ }^{15} \mathrm{O}$ reaction rate by the LUNA experiment (Formicola et al. 2004) and from the NACRE compilation (Angulo et al. 1999). The tracks obtained with the CF88 rate practically coincide with those obtained with the NACRE rate, as shown by Imbriani et al. (2004). The LUNA rate was simulated by simply multiplying that of Caughlan \& Fowler by a factor of 0.6 (LUNA Collaboration, Bemmerer et al. 2006; Imbriani et al. 2005; Lemut et al. 2006). The results are shown in Fig. 3 for $Z=0.004$ and $Y=0.26$, and in Fig. 4 for $Z=0.017$ and $Y=0.26$ for the masses indicated in the figures. Additional tracks for $Z=0.008$ were computed, but are not shown for conciseness. From these tracks, we conclude that:

- the MS becomes slightly bluer and slightly more luminous in models with a LUNA rate, as a consequence of the $\mathrm{H}$-burning lifetimes becoming shorter by about $1.5 \%$. This is in general agreement with the results by Weiss et al. (2005);

- the He-burning lifetimes do not change. He-burning loops have their $T_{\text {eff }}$ extension practically unchanged for $Z=0.004$ and 0.008 , whereas for $Z=0.017$ the loops become more extended with LUNA rates. This effect is surprising, and goes in the opposite sense to the results described in Weiss et al. (2005).

Overall, these changes in the tracks are very modest. Since the loops do not change at sub-solar metallicities, the comparison with Cepheid data are not affected. 


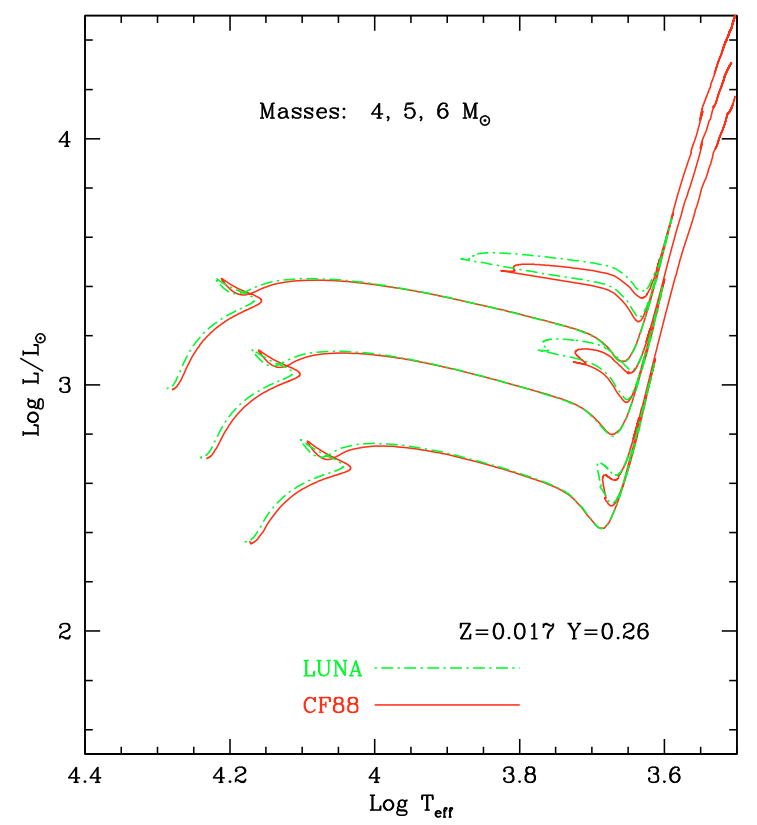

Fig. 4. Evolutionary tracks in the HR diagram for the composition $Z=0.017, Y=0.26$ computed with the new LUNA rate for the ${ }^{14} \mathrm{~N}(\mathrm{p}, \gamma){ }^{15} \mathrm{O}$ nuclear reaction (dot-dashed line) and with the rate according to Caughlan \& Fowler (1988) (solid line).

\subsection{Changes in surface chemical composition}

The surface chemical composition of the stellar models is affected by two defined dredge-up events. The first occurs on the first ascent of the RGB for all stellar models (except for the verylow mass ones which do not evolve beyond the main sequence). The second dredge-up is found after the core He-exhaustion, being remarkable only in models with $M \gtrsim 3.5 M_{\odot}$ and $M \lesssim 6 M_{\odot}$. For masses higher than $6 M_{\odot}$, the second dredge-up occurs in concomitance with the carbon ignition at the core, so that the exposition of He-enriched material at the surface is too shortlived to be of interest, and not followed in detail by the present calculations. We provide tables with the surface chemical composition of $\mathrm{H},{ }^{3} \mathrm{He},{ }^{4} \mathrm{He}$, and main $\mathrm{CNO}$ isotopes, before and after the first dredge-up, and after the second dredge-up. As an example, Table 2 shows the surface abundances for the chemical composition $Z=0.008$ and $Y=0.26$.

\section{Isochrones}

From the tracks presented in this paper, we constructed isochrones as in Paper I, with the algorithm of "equivalent evolutionary points" used in Bertelli et al. (1994) and Girardi et al. (2000). The initial point of each isochrone is the $0.15 M_{\odot}$ model in the lower main sequence. The terminal stage of the isochrone is the tip of the TP-AGB or the beginning of carbon burning. We note that there are usually small differences in the logarithm of the effective temperature between the last model of the track at the beginning of the TP-AGB and the starting point of the synthetic TP-AGB model of the corresponding mass. Constructing the isochrones, we removed these small discontinuities with a suitable shift.

A comparison of isochrones with the same metallicity $(Z=0.001)$ and different helium content $(Y=0.23$ and $Y=0.40$ ) can be found in Fig. 5 showing the range of variations in luminosity and temperature for some isochrones $(\log ($ Age $/ y r)=8.9,8.6,8.2,7.8,7.4,7.2)$. The extension to the end of the TP-AGB phase is also shown for each isochrone. An increase in the helium content at the same metallicity in stellar models causes a decrease in the mean opacity and an increase in the mean molecular weight of the envelope (Vemury $\&$ Stothers 1978), and in turn higher luminosities, hotter effective temperatures and shorter hydrogen and helium lifetimes of stellar models. In apparently conflict with the previous statement about higher luminosity for a helium increase in evolutionary tracks, the evolved portion of the isochrones with lower helium are more luminous, as shown in Fig. 5 where we plot isochrones with $Z=0.001$ for $Y=0.23$ and $Y=0.40$. This effect is related to the interplay between the increase in luminosity and the decrease in lifetime of stellar models with higher helium content (at the same mass and metallicity).

\subsection{Reliability of the interpolation}

The program YZVAR is used to both obtain isochrones and simulate stellar populations. The method of interpolation has been described in detail in Paper I. In the database, there are 37 sets of stellar tracks covering a large region of the $Z-Y$ plain and users can obtain either isochrones for whichever $Z-Y$ combination inside the provided range, or stellar populations with the required $Y(Z)$ enrichment law.

Most of the problems about the reliability of the interpolation originate from the extension of the loops during the central He-burning, which varies significantly from low to high metal content for the considered range of mass. For example, if we consider the two subsequent values of $Z$ of our grid, $Z=0.017$ and $Z=0.040$, we have extended loops for the first one (starting from a mass that increases with helium content $Y$ ). No loops are present for the upper mass $20 M_{\odot}$ for $Y>0.26$. In the case of $Z=0.040$, for all $Y$ values and masses, no loops are present, as shown in Fig. 2. In this case, we cannot expect the extension of the loops to have a linear behaviour as a function of the metal content in the interpolation for intermediate chemical compositions. We analysed the interpolation for $Z=0.0285$ and two intermediate cases, namely $Y=0.28$ and $Y=0.37$.

In the case of $Z=0.0285, Y=0.28$ the loops obtained by interpolation are significantly less extended and/or less luminous than those of tracks computed for the same chemical composition at 7 and $10 M_{\odot}$. In contrast, the interpolated tracks of 15 and $20 M_{\odot}$ exhibit significant loops, while in the computed ones there are no loops. In Fig. 6 the solid green lines represent the computed tracks, and the red dotted lines represent the interpolated tracks.

To improve the interpolation for this range of metal content, we computed new tracks with $Z=0.030, Y=0.26,0.30,0.34$, and 0.40 for $M>4.5 M_{\odot}$. In this, way the results of the interpolation become far more reliable, as evident in Fig. 6, where the blue line shows the result of the interpolation after taking into account this new set for masses greater than $4.5 M_{\odot}$. The comparison of the lifetimes of the computed and the interpolated tracks infers differences of lower than $3 \%$. The interpolation is also superior in the case of $Z=0.0285$ and $Y=0.37$, when taking into account the new tracks computed for the intermediate metal content $Z=0.030$.

Another critical region of the interpolation is at low metallicities (of $Z$ between 0.0004 and 0.001 for high values of $Y$ ) owing to the different morphological behaviour of the involved tracks. A computed set of tracks $\left(5,7,10,15,20 M_{\odot}\right)$ with $Z=0.0007$ and $Y=0.35$ was compared with a corresponding interpolated set and the results are shown in Fig. 7, where the green lines represent the computed tracks and the red dotted lines the 
Table 2. Surface chemical composition (by mass fraction) of $[Z=0.008, Y=0.26]$ models.

\begin{tabular}{|c|c|c|c|c|c|c|c|c|c|c|}
\hline$M / M_{\odot}$ & $\mathrm{H}$ & ${ }^{3} \mathrm{He}$ & ${ }^{4} \mathrm{He}$ & ${ }^{12} \mathrm{C}$ & ${ }^{13} \mathrm{C}$ & ${ }^{14} \mathrm{~N}$ & ${ }^{15} \mathrm{~N}$ & ${ }^{16} \mathrm{O}$ & ${ }^{17} \mathrm{O}$ & ${ }^{18} \mathrm{O}$ \\
\hline $\begin{array}{l}\text { Initial: } \\
\text { all }\end{array}$ & 0.732 & $2.78 \times 10^{-5}$ & 0.260 & $1.37 \times 10^{-3}$ & $1.65 \times 10^{-5}$ & $.24 \times 10^{-4}$ & & $3.85 \times 10^{-3}$ & $1.56 \times 10^{-6}$ & $8.68 \times 10^{-6}$ \\
\hline \multicolumn{11}{|c|}{ After the first dredge-up: } \\
\hline 2.50 & 0.699 & $2.02 \times 10^{-4}$ & 0.292 & $8.50 \times 10^{-4}$ & $4.40 \times 10^{-5}$ & $1.32 \times 10^{-3}$ & $8.18 \times 10^{-7}$ & $3.49 \times 10^{-3}$ & $1.05 \times 10^{-5}$ & $6.25 \times 10^{-6}$ \\
\hline 3.00 & 0.700 & $1.42 \times 10^{-4}$ & 0.292 & $8.48 \times 10^{-4}$ & $4.45 \times 10^{-5}$ & $1.36 \times 10^{-3}$ & $8.11 \times 10^{-7}$ & $3.44 \times 10^{-3}$ & $7.60 \times 10^{-6}$ & $6.24 \times 10^{-6}$ \\
\hline 3.50 & 0.702 & $1.07 \times 10^{-4}$ & 0.290 & $8.50 \times 10^{-4}$ & $4.46 \times 10^{-5}$ & $1.37 \times 10^{-3}$ & $8.10 \times 10^{-7}$ & $3.42 \times 10^{-3}$ & $6.13 \times 10^{-6}$ & $6.24 \times 10^{-6}$ \\
\hline 4.00 & 0.704 & $8.61 \times 10^{-5}$ & 0.287 & $8.62 \times 10^{-4}$ & $4.54 \times 10^{-5}$ & $1.35 \times 10^{-3}$ & $8.12 \times 10^{-7}$ & $3.43 \times 10^{-3}$ & $4.89 \times 10^{-6}$ & $6.32 \times 10^{-6}$ \\
\hline 4.50 & 0.707 & $7.16 \times 10^{-5}$ & 0.285 & $8.72 \times 10^{-4}$ & $4.49 \times 10^{-5}$ & $1.34 \times 10^{-3}$ & $8.16 \times 10^{-7}$ & $3.43 \times 10^{-3}$ & $4.23 \times 10^{-6}$ & $6.33 \times 10^{-6}$ \\
\hline 5.00 & 0.708 & $6.04 \times 10^{-5}$ & 0.284 & $8.85 \times 10^{-4}$ & $4.52 \times 10^{-5}$ & $1.33 \times 10^{-3}$ & $8.26 \times 10^{-7}$ & $3.43 \times 10^{-3}$ & $4.05 \times 10^{-6}$ & $6.37 \times 10^{-6}$ \\
\hline 6.00 & 0.710 & $4.70 \times 10^{-5}$ & 0.282 & $8.82 \times 10^{-4}$ & $4.74 \times 10^{-5}$ & $1.31 \times 10^{-3}$ & $8.09 \times 10^{-7}$ & $3.45 \times 10^{-3}$ & $3.46 \times 10^{-6}$ & $6.42 \times 10^{-6}$ \\
\hline 7.00 & 0.711 & $3.89 \times 10^{-5}$ & 0.281 & $8.81 \times 10^{-4}$ & $4.76 \times 10^{-5}$ & $1.32 \times 10^{-3}$ & $8.00 \times 10^{-7}$ & $3.45 \times 10^{-3}$ & $3.06 \times 10^{-6}$ & $6.40 \times 10^{-6}$ \\
\hline 8.00 & 0.711 & $3.35 \times 10^{-5}$ & 0.281 & $8.77 \times 10^{-4}$ & $4.84 \times 10^{-5}$ & $1.33 \times 10^{-3}$ & $7.90 \times 10^{-7}$ & $3.44 \times 10^{-3}$ & $3.02 \times 10^{-6}$ & $6.37 \times 10^{-6}$ \\
\hline 10.0 & 0.711 & $2.73 \times 10^{-5}$ & 0.281 & $8.75 \times 10^{-4}$ & $4.91 \times 10^{-5}$ & $1.33 \times 10^{-3}$ & $7.75 \times 10^{-7}$ & $3.44 \times 10^{-3}$ & $3.10 \times 10^{-6}$ & $6.33 \times 10^{-6}$ \\
\hline 12.0 & 0.703 & $2.34 \times 10^{-5}$ & 0.289 & $8.65 \times 10^{-4}$ & $5.03 \times 10^{-5}$ & $1.42 \times 10^{-3}$ & $7.56 \times 10^{-7}$ & $3.34 \times 10^{-3}$ & $2.71 \times 10^{-6}$ & $6.19 \times 10^{-6}$ \\
\hline 15.0 & 0.683 & $1.95 \times 10^{-5}$ & 0.309 & $8.30 \times 10^{-4}$ & $5.01 \times 10^{-5}$ & $1.64 \times 10^{-3}$ & $7.16 \times 10^{-7}$ & $3.15 \times 10^{-3}$ & $2.24 \times 10^{-6}$ & $5.86 \times 10^{-6}$ \\
\hline 20.0 & 0.731 & $2.54 \times 10^{-5}$ & 0.261 & $1.23 \times 10^{-3}$ & $6.69 \times 10^{-5}$ & $5.49 \times 10^{-4}$ & $1.00 \times 10^{-6}$ & $3.83 \times 10^{-3}$ & $1.60 \times 10^{-6}$ & $8.42 \times 10^{-6}$ \\
\hline \multicolumn{11}{|c|}{ After the second dredge-up: } \\
\hline 3.50 & 0.693 & $1.03 \times 10^{-}$ & 0.298 & & & & & $3.37 \times 10^{-3}$ & $6.98 \times 10^{-6}$ & $6.06 \times 10^{-6}$ \\
\hline 4.00 & 0.675 & $8.00 \times 10^{-5}$ & 0.317 & $8.08 \times$ & $4.48 \times$ & $1.55 \times 1$ & $7.56 \times 1$ & $3.28 \times 10^{-3}$ & $5.60 \times 10^{-6}$ & $5.93 \times 10^{-6}$ \\
\hline 4.50 & 0.658 & $6.44 \times$ & 0.334 & $7.95 \times$ & $4.40 \times$ & $1.64 \times 1$ & $7.40 \times 1$ & $3.19 \times 10^{-3}$ & $4.76 \times 10^{-6}$ & $5.77 \times 10^{-6}$ \\
\hline 5.00 & 0.644 & $5.27 \times 10^{-5}$ & 0.348 & $7.85 \times 10^{-4}$ & $4.39 \times 10^{-5}$ & $1.72 \times 10^{-3}$ & $7.29 \times 10^{-7}$ & $3.12 \times 10^{-3}$ & $4.45 \times 10^{-6}$ & $5.63 \times 10^{-6}$ \\
\hline
\end{tabular}

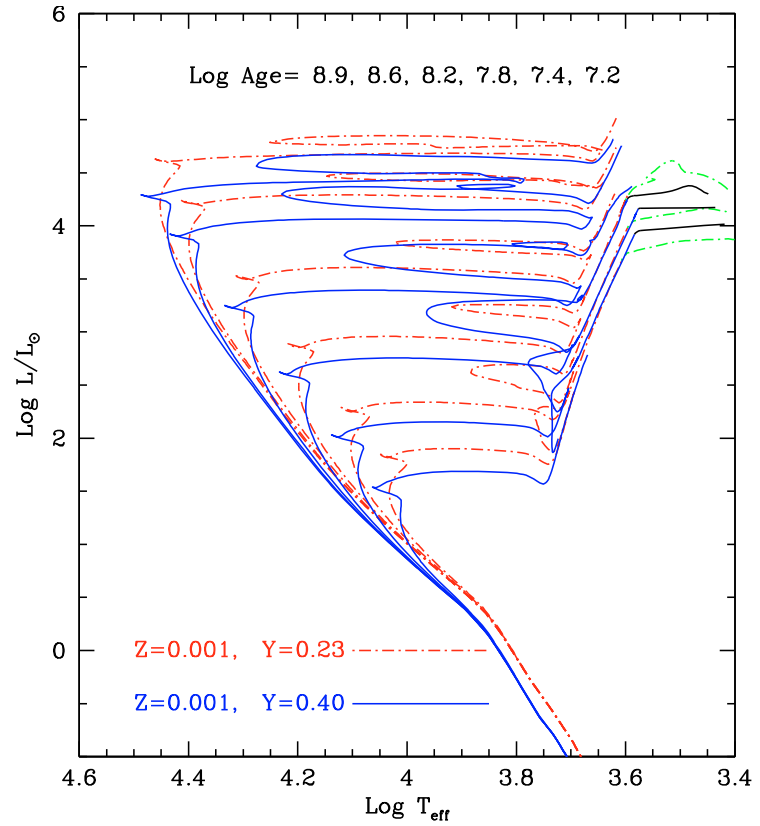

Fig. 5. Comparison of isochrones for the same $Z=0.001$ and different helium content. Blue solid lines correspond to $Y=0.40$ (the related AGB is in black), and red dot-dashed ones to $Y=0.23$ (the related AGB is in green).

interpolated ones. There are some differences in the red region, which is reached for the first time after the central hydrogen exhaustion for the 7 and $10 M_{\odot}$. If we check the lifetime of the phases at variance with the computed tracks (see Fig. 8), the differences are negligible with respect to the total He-burning lifetime.

In all other cases, the comparisons are satisfactory.

\subsection{Bolometric corrections}

The present isochrones are provided in the Johnson-CousinsGlass system as defined by Bessell (1990) and Bessell \& Brett (1988), in the Vegamag systems of ACS onboard HST (cf.

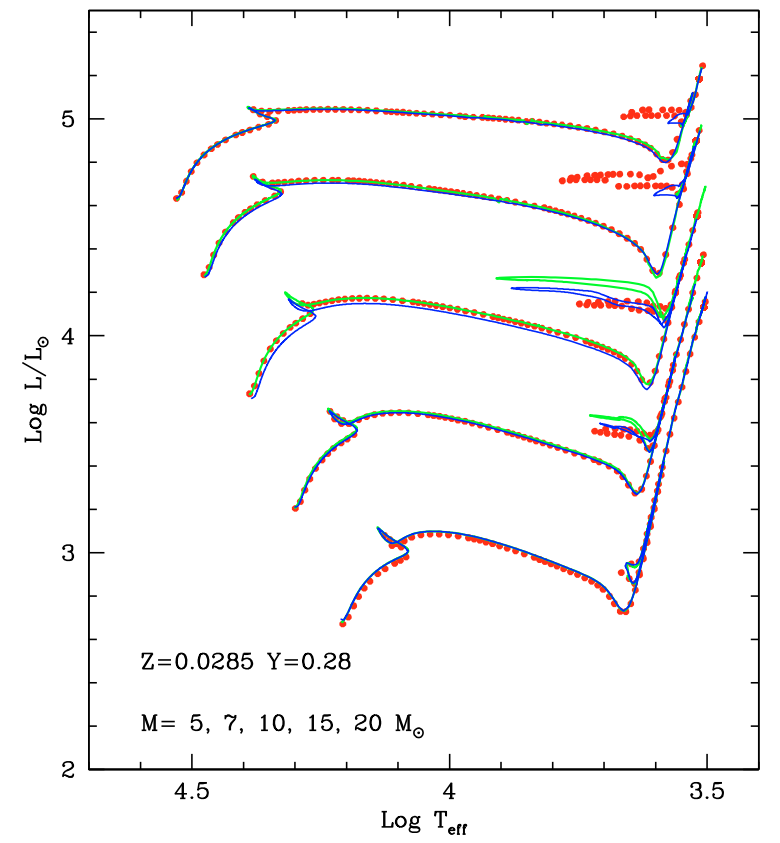

Fig. 6. Comparison between interpolated and computed tracks with $Z=0.0285, Y=0.28$. The red dotted lines are the interpolated tracks between $Z=0.017$ and $Z=0.040$, while the green solid lines are the computed ones for the specific $Z$. The blue lines are the interpolated tracks taking into account the new set computed for $Z=0.030$ and various $Y$. Taking into account the new set, the interpolation is in closer agreement with the actual computed tracks.

Sirianni et al. 2005), and in the SDSS system. The formalism that we follow to derive bolometric corrections in these systems is described in Girardi et al. (2002). The definition of zeropoints was revised as described in a paper by Girardi et al. (2008), a description that is not repeated here.

Suffice it to say that the bolometric correction tables are based on an updated and extended library of stellar spectral fluxes. The core of the library now consists of the "ODFNEW" 


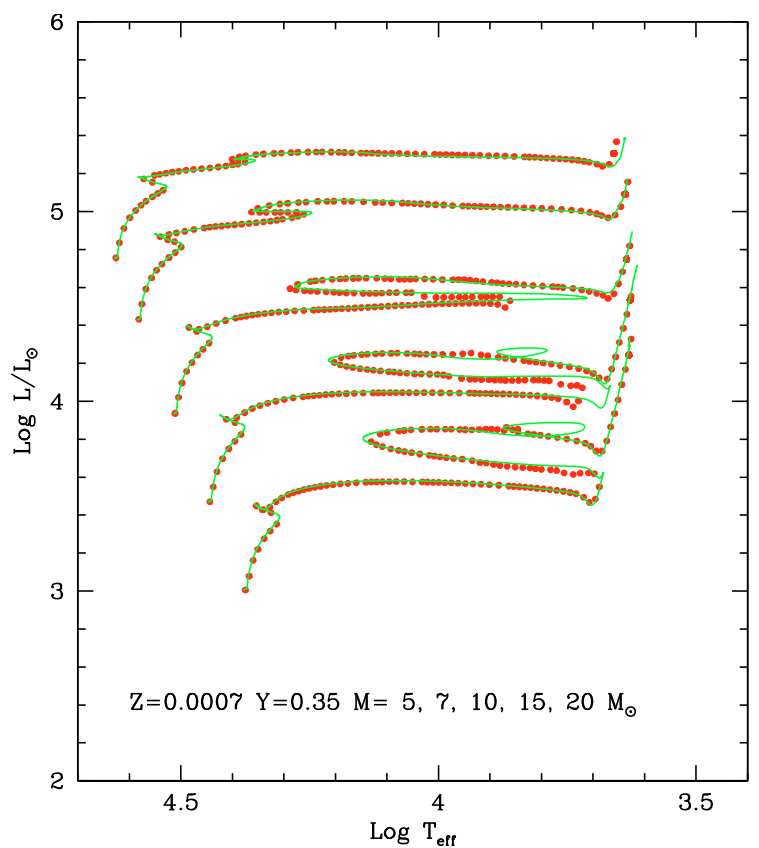

Fig. 7. Comparison between interpolated and computed tracks with $Z=$ $0.0007, Y=0.35$. The red dotted lines are the interpolated tracks for masses between 5 and $20 M_{\odot}$ and the green solid lines the computed lines for the specific $Z$.
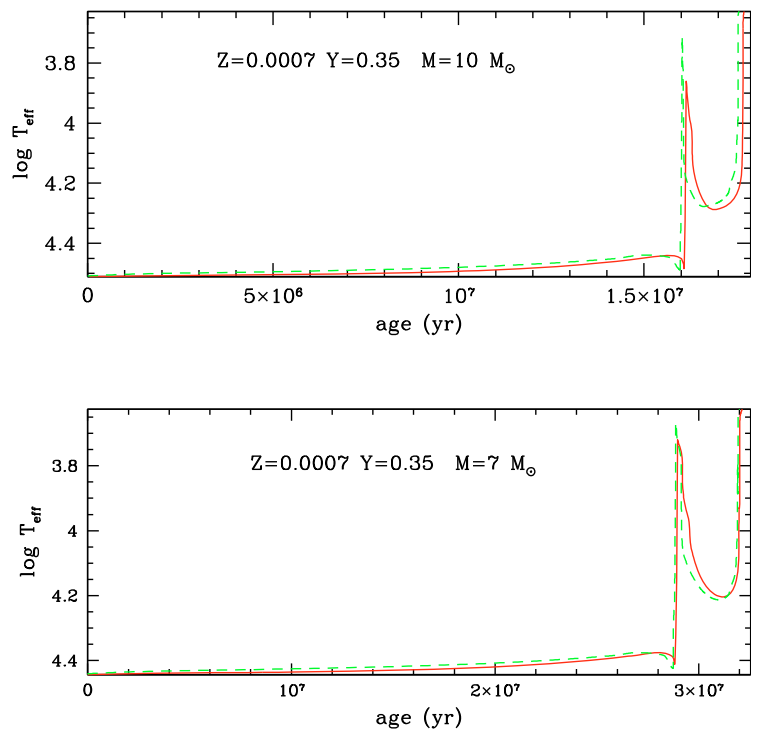

Fig. 8. Differences between the lifetime spent by the interpolated track (red solid line) and that of the computed track (green dashed line) are insignificant. The chemical composition is as in Fig. 7.

ATLAS9 spectral fluxes from Castelli \& Kurucz (2003), for $T_{\text {eff }}$ of between 3500 and $50000 \mathrm{~K}, \log g$ of between -2 and 5 , and scaled-solar metallicities $[\mathrm{M} / \mathrm{H}]$ of between -2.5 and +0.5 . This library is extended to high $T_{\text {eff }}$ by using pure black-body spectra. For lower $T_{\text {eff }}$, the library is completed with spectral fluxes for M, L, and T dwarfs from Allard et al. (2000), M giants from Fluks et al. (1994), and finally C star spectra from Loidl et al. (2001). Details about the implementation of this library, and in particular about the $\mathrm{C}$ star spectra, are provided in Marigo et al. (2008) and Girardi et al. (2008).

We note that in the isochrones we apply the bolometric corrections derived from this library without making any correction for the enhanced He content. As demonstrated in Girardi et al. (2007), for a given metal content, an enhancement of He as high as $\Delta Y=0.1$ produces changes in the bolometric corrections of only a few thousandths of magnitude. Just in some particular situations, for instance at low $T_{\text {eff }}$ and for blue passbands, the He-enhancement can produce more sizeable effects on BCs; these situations, however, correspond to cases where the emitted stellar flux would in all cases be very small, and therefore are of little interest.

\subsection{Description of isochrone tables}

Complete tables with the isochrones can be obtained through our web site ${ }^{2}$ interactive services.

The user must select the chemical composition, the $\Delta \log t$ spacing between contiguous isochrones, and the photometric system. The following photometric systems are available:

- UBVRIJHK absolute magnitudes in the Johnson-CousinsGlass photometric system.

- ACS-WFC photometric system (bolometric corrections from Girardi et al. 2008). In the tables, the absolute magnitudes for the following 12 filters are listed: $F 435 W, F 475 W, F 550 M$, F555W, F606W, F625W, F658N, F660N, FF775W, F814W, F850LP, F892N.

- ACS-HRC photometric system (see Girardi et al. 2008). The absolute magnitudes listed in the tables are for the following 16 filters: $F 220 \mathrm{~W}, \mathrm{~F} 250 \mathrm{~W}, \mathrm{~F} 330 \mathrm{~W}, \mathrm{~F} 344 \mathrm{~N}, \mathrm{~F} 435 \mathrm{~W}, \mathrm{~F} 475 \mathrm{~W}$, F550M, F555W, F606W, F625W, F658N, F660N, F775W, F814W, F850LP, F892N.

In the case of the Johnson-Cousins-Glass system, the isochrone tables contain the following information:

1. $\log A g e: \log$ arithm of the age in years;

2. $\log (\mathrm{L} / \mathrm{Lo}): \operatorname{logarithm}$ of surface luminosity (in solar units);

3. logTef: logarithm of effective temperature (in K);

4. logG: logarithm of surface gravity (in cgs units);

5. Mi: initial mass in solar masses;

6. Mcur: actual stellar mass in solar masses;

7. FLUM: indefinite integral over the initial mass $M$ of the Salpeter initial mass function by number;

8. - 15.: UBVRIJHK absolute magnitudes in the JohnsonCousins-Glass system;

16. $\mathrm{C} / \mathrm{O}$ the surface $\mathrm{C} / \mathrm{O}$ ratio;

17. C.P.: index marking the presence of a characteristic point, when different from zero.

The isochrone tables for the ACS-WFC photometric system contain the same information in the first 7 columns, then the absolute magnitudes for this system from Cols. 8 to 19, and the last two columns are the same as in the previous case. For the ACS-HRC photometric system from Cols. 8 to 23 the absolute magnitudes for the relative filters are presented.

We note that the initial mass is the useful quantity for population synthesis calculations, since together with the initial mass function it determines the relative number of stars in different sections of the isochrones. In Col. 7, the indefinite integral evaluated over the initial mass $M$ of the initial mass function (IMF) by number, i.e.,

FLUM $=\int \phi(M) \mathrm{d} M$

${ }^{2}$ http://stev.oapd.inaf.it/YZVAR 
is presented, for the case of the Salpeter IMF, $\phi(M)=A M^{-\alpha}$, were $\alpha=2.35$. Setting $A=1$, FLUM is simply given by FLUM $=M^{1-\alpha} /(1-\alpha)$. This is a useful quantity since the difference between any two values of FLUM is proportional to the number of stars located in the corresponding isochrone interval. For example, if we consider an old isochrone fitting the observations of a globular cluster, and along the RGB we consider three points identifying two intervals (1-2 and 2-3), the ratio of the differences of FLUM at each interval borders gives exactly the expected ratio of the number of stars in the corresponding region of the observed globular cluster, as shown in the relation:

$\left(\mathrm{FLUM}_{3}-\mathrm{FLUM}_{2}\right) /\left(\mathrm{FLUM}_{2}-\mathrm{FLUM}_{1}\right)=N_{2-3}^{*} / N_{1-2}^{*}$,

where the terms have their usual meaning.

We remark that the Salpeter (1955) IMF is not valid for masses lower than about $1 M_{\odot}$. The user can easily derive FLUM relations for alternative choices of the IMF, by using the values of the initial mass that we present in Col. 5 of the isochrone tables. Finally, the last column may signal the presence of a characteristic evolutionary point. Values of 1 refer to points from the ZAMS to the early AGB phase, and values of 2 represent the TP-AGB phase. Along the TP-AGB, we mark its beginning, its end, and the point where $\mathrm{C} / \mathrm{O}$ increases above unity, corresponding to the transition from $\mathrm{M}$ to carbon stars, if present. The presence of only one characteristic point of type 2 indicates a very short TP-AGB phase.

\section{Cepheids}

From an evolutionary perspective, Cepheids are post-red giant stars crossing the instability strip on so-called blue loops following the onset of core He-burning. Obviously the extension of the loop and the distribution of the lifetime along it determines the appearance and the distribution of the Cepheids in the HR diagram. Many factors can modify the loops, including all those able to change the relative contribution to the total luminosity from the H-burning shell and the central He-burning core. In this respect, a crucial effect is produced by the physical treatment of the central and envelope convection and related uncertainties. For the intermediate-mass stars, appreciable core overshooting during the main sequence causes a smaller loop, while envelope overshooting during the RGB phase tends to favour more extended loops. The parameters characterizing the core and envelope overshoot in Padova models were calibrated with observational constraints (Alongi et al. 1991, 1993 and ref. therein). In Figs. 9-11, we present tracks for 2.5, 3, 4, 5, 6, 7, 8, and $10 M_{\odot}$ for values of the metallicity $Z=0.002, Z=0.004$, and $Z=0.008$. To compare the extension of the loops, in the same figures we plotted the corresponding tracks computed by the Teramo group (TE04, Pietrinferni et al. 2004). These TE04 stellar models have an upper mass limit of $10 M_{\odot}$ in their database. The main-sequence phases and the contraction towards the Hayashi limit are very similar for the two evolutionary grids. Our red giant phase appears slightly redder because of a different choice of mixing length parameter. The loops have different extensions, but this is normal because of the sensitivity of the loops to many of the input parameters.

The only true and puzzling difference is that related to the absence of loops in TE04 tracks at lower mass in the case $Z=$ 0.004. Our tracks illustrate a typical behaviour, in the sense that the minimum mass exhibiting a significant loop (able to intercept the instability strip of Cepheids) tends to increase with the metal content $Z$ (and with the helium content $Y$ at fixed metallicity).

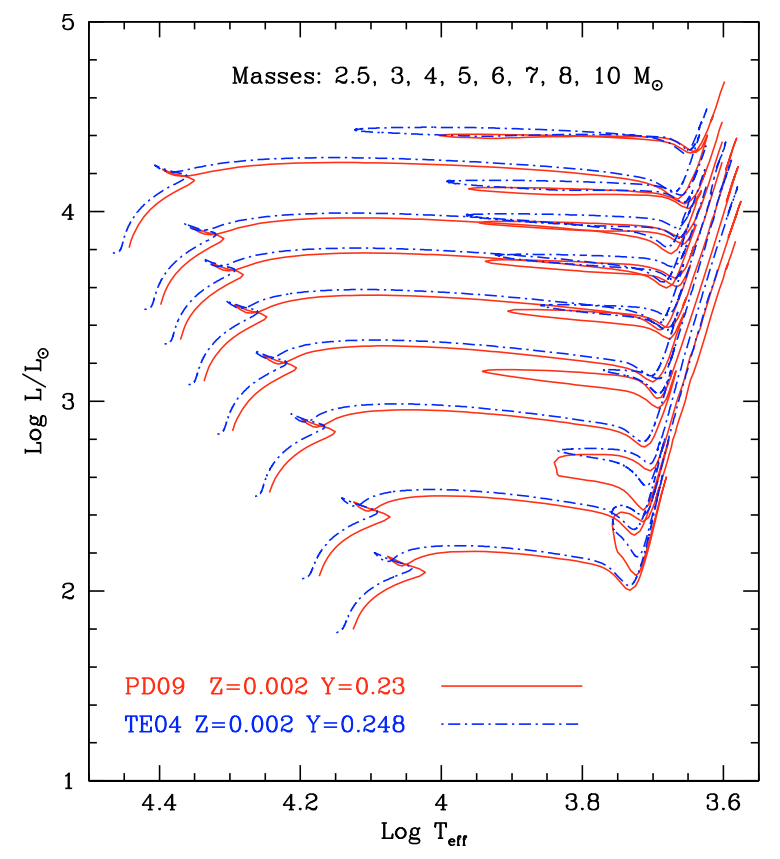

Fig. 9. PD09 evolutionary tracks with $Z=0.002, Y=0.23$ for masses between 2.5 and $10 M_{\odot}$ (solid line). TE04 models with $Z=0.002, Y=$ 0.248 (dot-dashed line) for the same mass range.

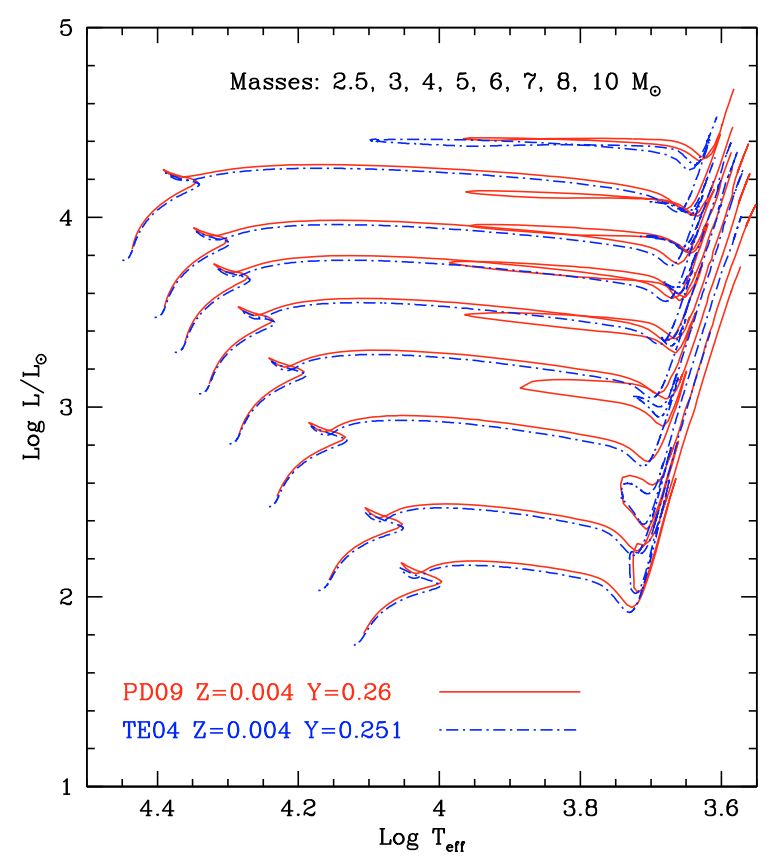

Fig. 10. PD09 evolutionary tracks with $Z=0.004, Y=0.26$ for masses between 2.5 and $10 M_{\odot}$ (solid line). TE04 models with $Z=0.004, Y=$ 0.251 (dot-dashed line) for the same mass range. It is evident in TE04 models the absence of loops for masses lower than $10 M_{\odot}$.

This prediction is confirmed by all the chemical compositions considered in our computations.

In contrast, the case $Z=0.004$ of TE04 appears to contradict this pattern, since only the $10 M_{\odot}$ model exhibits an extended loop, while for $Z=0.002$ and $Z=0.008$ loops are present at lower masses. This is at variance with the period (mass) distribution of Cepheids in the SMC, for which the metal content $Z=0.004$ is usually adopted.

A similar difference was already shown by evolutionary tracks of intermediate-mass stars with no convective 


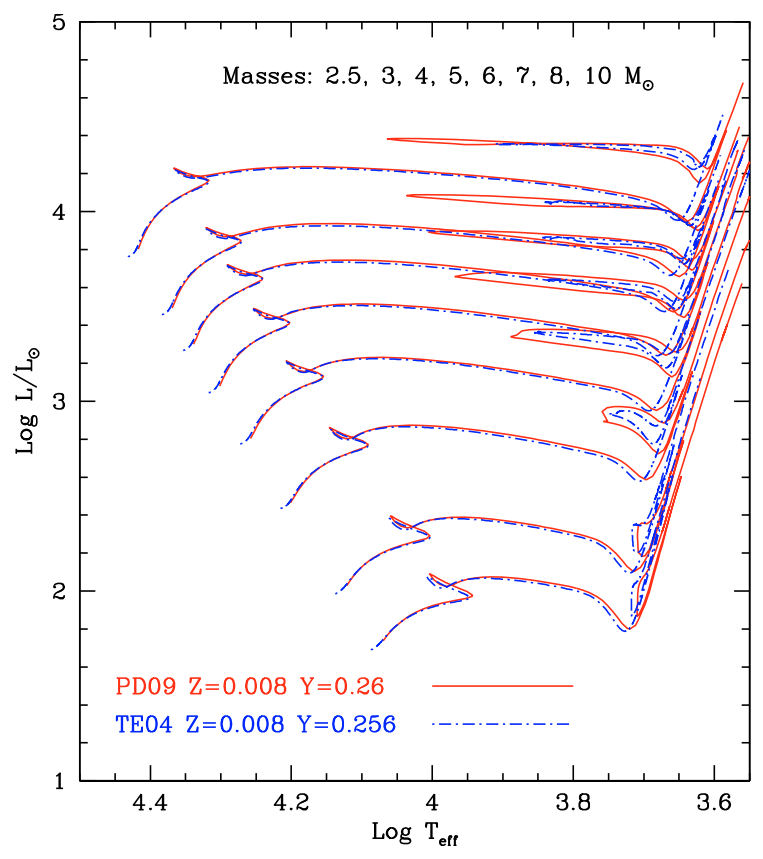

Fig. 11. PD09 evolutionary tracks with $Z=0.008, Y=0.26$ for masses between 2.5 and $10 M_{\odot}$ (solid line). TE04 models with $Z=0.008, Y=$ 0.256 (dot-dashed line) for the same mass range.

overshooting discussed in Castellani et al. (1990). They explained the decrease in the extension of the blue loop for masses around $5 M_{\odot}$ and the intermediate metal content $Z=0.006$ and $Y=0.23$, connected with the occurrence of the first dredge-up. It is not obvious why this feature is not present for higher $Y$ values and/or different $Z$, as would be expected.

\subsection{Comparison with the observations: $S M C, L M C$, Milky Way}

Cordier et al. (2003) explored the distribution of SMC Cepheids in the HR diagram in relation to the extension of the loops of the evolutionary tracks. They showed that the blue loops are sufficiently extended only if the metallicity is substantially lower than the usually adopted value for the SMC. They found that tracks with metallicity $Z=0.001$ correctly reproduce the boundary of the Cepheids towards lower luminosity. According to Cordier et al. (2003), Cepheids populating the faint bottom of the instability strip are likely to be metal-poor stars, while variables distributed throughout the instability strip should belong to populations of various metal contents.

Romaniello et al. (2008) derived direct measurements of the iron abundances of Galactic and Magellanic Cepheids from FEROS and UVES high-resolution and high signal-to noise spectra. The mean iron abundance $([\mathrm{Fe} / \mathrm{H}])$ was found to be about solar for the Galactic sample, with a range of values between $-0.18 \mathrm{dex}$ and $+0.25 \mathrm{dex}$. For the LMC sample, the mean value is about -0.33 dex with a range of values between -0.62 dex and -0.10 dex. For the SMC sample, the mean value is about -0.75 dex with a range of values between -0.87 and -0.63 dex.

If the behaviour of the loops in the evolutionary models is correct (i.e., the increase of the minimum mass that intercepts the instability strip with $Z$ and $Y$ ), this means that the lower limit to data for Cepheids in the HR diagram is determined by the stars of lower metal content $Z$. In Figs. 12-14 we checked whether our models can reproduce the lower boundaries of the instability

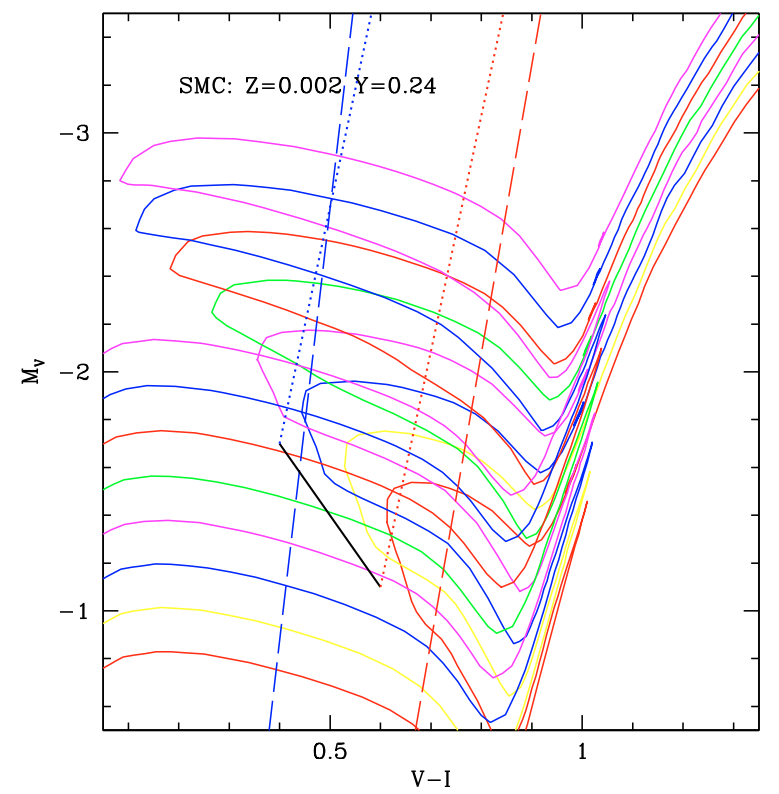

Fig. 12. PD09 isochrones with $Z=0.002, Y=0.24$ for ages in the range $8.30 \leq \log (t / \mathrm{yr}) \leq 8.65$. Long-dashed lines represent the blue and red edges of the theoretical instability strip (from Caputo et al. 2004). The dotted lines are the observed boundaries, and the black line represents the bottom of the instability strip from observations of SMC Cepheids (as in Fig. 7 by Sandage et al. 2009).

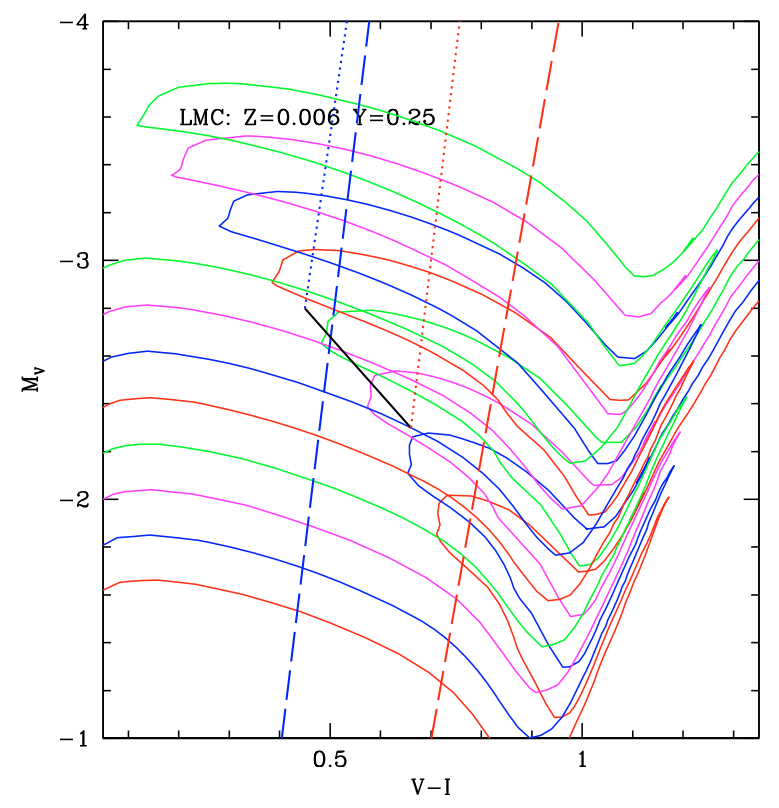

Fig. 13. PD09 isochrones with $Z=0.006, Y=0.25$ for ages in the range $8.05 \leq \log (t / \mathrm{yr}) \leq 8.40$. Long-dashed lines represent the blue and red edges of the theoretical instability strip (from Caputo et al. 2004). The dotted lines are the observed boundaries, and the black line represents the bottom of the instability strip from observations of LMC Cepheids (from Fig. 8 in Sandage et al. 2004).

strip compatibly with the dispersion in $Z$ found by Romaniello et al. (2008) for SMC, LMC, and MW respectively.

In Fig. 12 we show the loops of isochrones with ages in the range $8.30 \leq \log (t / \mathrm{yr}) \leq 8.65$ (the separation between the isochrones is $\Delta \log (t / y r)=0.05)$ and chemical composition $Z=0.002, Y=0.24$. The blue and red long dashed lines represent, respectively, the blue and red edges of the theoretical 
instability strip as obtained by Eqs. (1a) and (2a) in Caputo et al. (2004), where we adopt the mass-luminosity relation calibrated in our models. The black thick line represents the lower boundary of the instability strip from Fig. 7 of the paper on the SMC Cepheids by Sandage et al. (2009). The blue and red dotted lines are the experimental boundaries of the strip shown in the same Fig. 7 of Sandage et al. We note that:

- the observed instability strip is narrower than the theoretical one;

- the slope of the lower boundary for observed stars coincides with the slope of the faint envelope of the loops;

- the masses of the stars developing loops close to the observed lower boundary are in the range $2.5-3 M_{\odot}$;

- the observed lower limit could be recovered by models by adopting a slightly lower metal content than the assumed $Z=$ 0.002 (as given by Romaniello et al. 2008).

In Fig. 13, we plot the results for the Cepheids of the LMC. In this case, the chemical composition adopted for the set of isochrones is $Z=0.006, Y=0.25$. The bottom of the instability strip was taken from Fig. 8 of the paper on the LMC Cepheids by Sandage et al. (2004). The blue and red dotted lines correspond to the blue and red boundaries of the instability strip, such that the strip contains $\sim 90 \%$ of the Cepheids (Fig. 8 of Sandage et al. 2004). The blue and red long-dashed lines are the boundaries of the theoretical strip as represented by the formulae of Caputo et al. (2004) for the appropriate chemical composition. We point out that

- in the case of the LMC, the observed strip is also narrower than the theoretical one (the red edges differ significantly in position but not in slope);

- the ages of the loops shown are in the interval $8.05 \leq$ $\log (t / \mathrm{yr}) \leq 8.40$ (with $\Delta \log (t / \mathrm{yr})=0.05)$ and the masses of models with loops close to the observed bottom are in the range 3.7-4.4 $M_{\odot}$;

- the chemical composition adopted in our analysis $(Z=$ 0.006 ) that can reproduce the observations is $\sim 40 \%$ higher than the lower limit indicated by Romaniello et al. $(Z=$ $0.004)$ and $\sim 28 \%$ lower than the mean value $(Z=0.008)$, which is usually adopted for the LMC.

In the case of the Galaxy, we considered the calibration of Cepheids in open clusters and associations listed in Table 3 of the paper by Tammann et al. (2003). In Fig. 14 we plotted this sample of Cepheids over a set of isochrones with metal content $Z=0.010$ and $Y=0.26$ (this value of the metallicity corresponds to the lower limit of metallicity for the Galactic Cepheids as given in Romaniello et al. 2008). The observed instability strip is reproduced using Eqs. (5) and (16), and Fig. 15 of Tammann et al. (2003). Because of the small number of objects, it is impossible to draw the lower boundary of the observed strip as done for the LMC and the SMC. The only conclusion is that the value $Z=0.010$ is compatible with the data in the sense that the observed object with the highest magnitude lies very close to the faint envelope detected by the loops of the isochrones shown in Fig. 14.

\subsection{Mass discrepancy}

A long-standing open question is given by the discrepancy between the pulsation and the evolutionary mass of the Cepheids (for a brief review see Keller 2008). Caputo et al. (2005) considered 34 Cepheids in the Milky Way with solar-like metal content previously studied by Storm et al. (2004). They determined

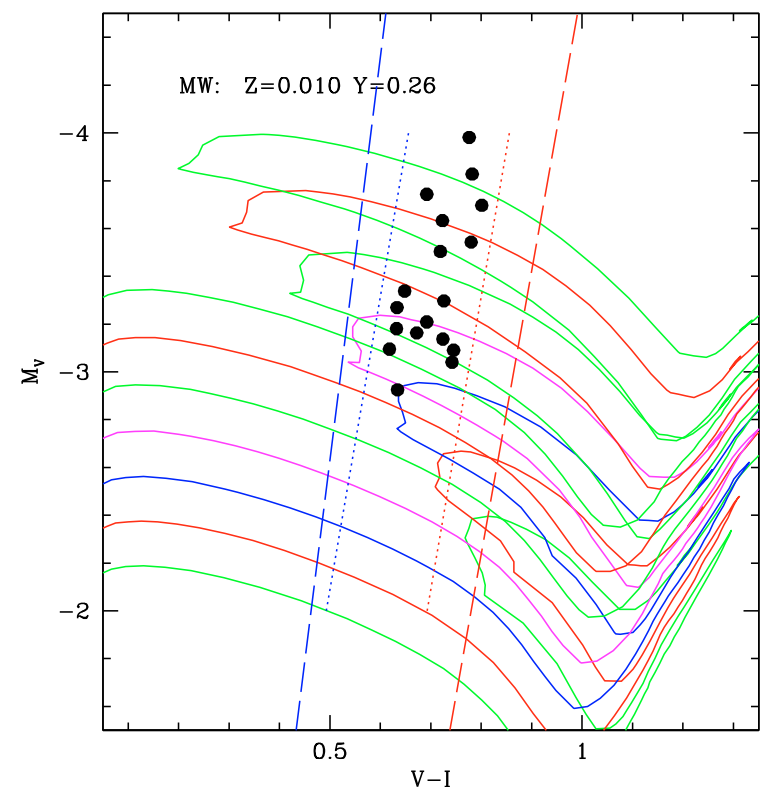

Fig. 14. PD09 isochrones with $Z=0.010, Y=0.26$ for ages in the range $7.95 \leq \log (t / \mathrm{yr}) \leq 8.25$. Long-dashed lines represent the blue and red edges of the theoretical instability strip (from Caputo et al. 2004). The dotted lines are the observed boundaries, and the Cepheids in open clusters and associations are plotted (from Tammann et al. 2003).

the pulsation mass $M_{\mathrm{p}}$ from the predicted PLC relations and the evolutionary one $M_{\mathrm{e}}$ from the mass-period-luminosity (MPL) or the mass-color-luminosity (MCL) relations adopting the canon$\mathrm{ical}^{3}$ relation mass-luminosity (ML). The comparison between the two determinations of the mass showed that the $M_{\mathrm{p}} / M_{\mathrm{e}}$ ratio is correlated with the Cepheid period, ranging from $\sim 0.8$ at $\log (P /$ days $)=0.5$ to $\sim 1$ at $\log (P /$ days $)=1.5$. In other words, the discrepancy between the pulsation and the evolutionary mass decreases when moving from lower to higher mass Cepheids and disappears for masses around $13-14 M_{\odot}$. The authors interpreted this discrepancy as an effect of mass loss occurring during and before the central He-burning phase. However, the data would imply a mass-loss efficiency that decreases with increasing Cepheid initial mass; this mass-loss is at odds with empirical estimates where the mass loss increases with stellar luminosity and radius (Schroeder \& Cuntz 2007). Another plausible solution of the Cepheid mass discrepancy is given by extra convective mixing; in this case, the relation ML is modified because the luminosity of the He-burning phase is higher than in the canonical case and increases with an increasing amount of extra mixing. Chiosi et al. (1992a,b) discussed the mass discrepancy of Cepheids by comparing models incorporating either semiconvection or overshoot and determining the pulsation mass from Cepheid models (Chiosi et al. 1993). In the case of models with overshoot, the discrepancy no longer exists for the considered Cepheids in the young rich LMC cluster NGC 2157.

On the one side, mass loss, in an ad-hoc manner at least, offers a mechanism capable of modifying the M-L relation by directly reducing the mass of a Cepheid. On the other side, according to a discussion by Neilson \& Lester (2008, 2009), considering winds driven not only by radiation, but including momentum input from pulsation and shocks generated in the atmosphere, the calculated mass-loss rate for Cepheids is enhanced

\footnotetext{
3 By this term, we mean without overshooting from the convective core.
} 


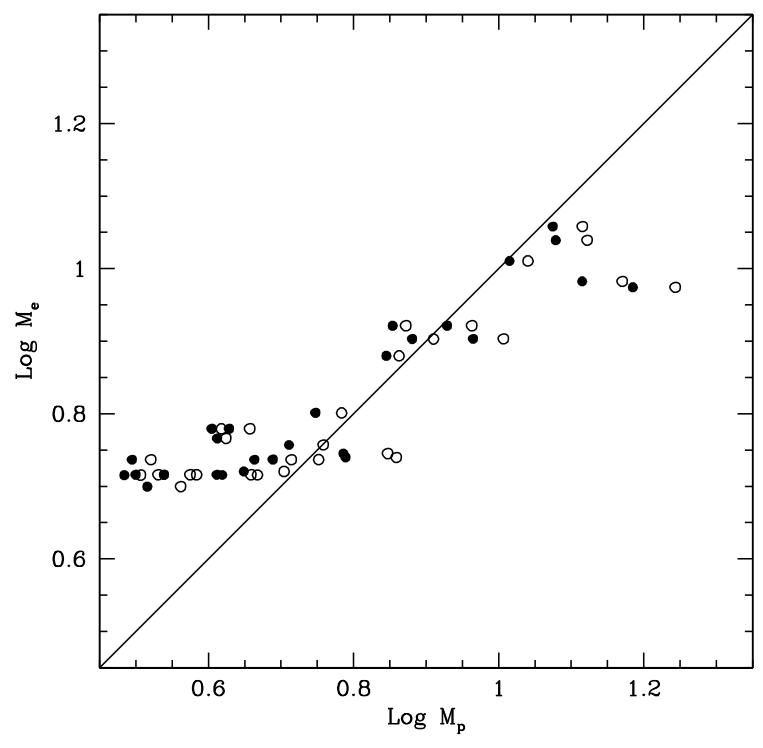

Fig. 15. Cepheids in open clusters and associations (from the paper by Tammann et al. 2003) are plotted with the pulsation and the evolutionary mass determined as described in the text. Solid dots are pulsation masses determined according to the PLC relation by Caputo et al. (2005) and open circles according to the PLC relation by Chiosi et al. (1993).

and helps us to illuminate the issue of infrared excess and the mass discrepancy,

After reanalysing the results of Caputo et al. (2005), Keller (2008) rejects the mass loss hypothesis and reaches the conclusion that an increased internal mixing remains the most likely cause of the Cepheids mass discrepancy.

As an exercise, we considered the same sample of Cepheids used in Fig. 14 (Cepheids in open clusters of Table 3 of Tammann et al. 2003). For a solar chemical composition $(Z=$ $0.017, Y=0.27$ ) we computed a dense grid of isochrones $(\Delta \log (t / y r)=0.05)$, then for each point (Cepheid) of the plane $M_{V}-\left(M_{V}-M_{I}\right)$, we obtained the minimum distance from the points which describe the totality of the isochrones. This minimum singles out the age and the evolutionary mass $M_{\mathrm{e}}$ of the Cepheid under consideration (with an uncertainty related to the ranging of isochrones). The corresponding pulsation mass $M_{\mathrm{p}}$ was computed using the mass-dependent PLC relation for fundamental pulsators (employing the observed quantities $M_{I}$ and $\left(M_{V}-M_{I}\right)$ of Table 2 in Caputo et al. 2005). The estimated pulsation and evolutionary masses are presented in Fig. 15. In the same figure, the open symbols represent the pulsation masses computed with the PLC relation by Chiosi et al. (1993) (we point out that the opacity tables used in Chiosi et al. differ from those of Caputo et al. and those of the present paper).

Independently from the formulation used to obtain $M_{\mathrm{p}}$, the data plotted in this figure show that for stars of mass lower than $\sim 6 M_{\odot}$, the evolutionary mass is higher than the pulsation one $\left(M_{\mathrm{e}}>M_{\mathrm{p}}\right)$, while for stars more massive than $10 M_{\odot}$ the pulsation mass is higher than the evolutionary one $\left(M_{\mathrm{e}}<M_{\mathrm{p}}\right)$. In the range $6-10 M_{\odot}$, the parametrization $\Lambda_{\mathrm{c}}=0.5$ could be satisfactory. We could interpret this by suggesting that the parameter $\Lambda_{c}=0.5$, which determines the efficiency of the extramixing, must be a function of the mass of the star. In other words, $\Lambda_{\mathrm{c}}$ should decrease with increasing stellar mass, assuming values close to $\Lambda_{c}=0.5$ in the range $6-10 M_{\odot}$. This should be taken as a suggestion and not a firm result because the number of stars in the sample is very limited; the values of the absolute magnitudes of the Cepheids are significantly different depending on the different estimates.

From our sample, we excluded GY Sge and S Vul because their $M_{\mathrm{p}}$ was $>20 M_{\odot}$ (i.e., beyond the validity range of theoretical relations), and EV Sct because it is probably a binary. The differences between the results of Keller and ours are mainly caused by Keller proposing an additional internal mixing, parametrized by $\Lambda_{c}=0.67 \pm 0.17$ for the whole mass range, and us suggesting an extra mixing that decreases with the stellar mass, but with $\Lambda_{\mathrm{c}} \approx 0.5$ in the range $6-10 M_{\odot}$.

\section{Massive red supergiants}

Red supergiants (RSGs) are a He-burning phase in the evolution of moderately high mass stars $\left(10-25 M_{\odot}\right)$. The evolution of these stars, particularly at low metallicities, is still poorly understood. The latest-type RSGs in the Magellanic Clouds are cooler than the current evolutionary tracks allow, occupying the region to the right of the Hayashi limit where stars are no longer in hydrostaic equilibrium. Until recently, the location of Galactic red supergiants (RSGs) in the Hertzsprung-Russell (H-R) diagram was poorly matched by stellar evolutionary tracks, evolutionary theory being unable to produce stars as cool and luminous as those observed (Massey 2003). Many possible explanations might contribute to this discrepancy: there is poor knowledge of RSG molecular opacities, the near-sonic velocities of the convective layers invalidate simplifications of mixing length theory, and the highly extended atmospheres of these stars differ from the plane-parallel geometry assumption adopted by evolutionary models (Levesque et al. 2006). A similar problem was evident for RSGs in the Magellanic Clouds (MCs) as shown by the data of Massey \& Olsen (2003) with the more robust available calibration at that time.

Afterwards Levesque et al. (2005, 2006) used moderateresolution optical spectrophotometry and the new MARCS stellar atmosphere models (Plez 2003; Gustafsson et al. 2003) to determine the physical properties of RSGs in both the Milky Way and the MCs for the comparison with the evolutionary models. They derived a new effective temperature scale significantly warmer than those in the literature, showing that the newly derived temperatures and bolometric corrections provide closer agreement with stellar evolutionary tracks. The comparison of Galactic RSGs was with the Geneva evolutionary tracks of solar metallicity (Meynet \& Maeder 2003) spanning a range of initial rotational velocities from 0 to $300 \mathrm{~km} \mathrm{~s}^{-1}$. Levesque et al. (2006) extended their study to Magellanic Cloud red supergiants to test the effects of metallicity and found evidence of significant visual extinction because of circumstellar dust. The effective temperatures of K supergiants are about the same in the SMC, LMC and Milky Way but the lower abundance of TiO leads to effective temperatures that are lower for M supergiants of the same spectral subtype. On average, RSGs in the Magellanic Clouds are not as cool as Galactic RSGs, in agreement with the shifting of the rightmost extension (the Hayashi limit) of the evolutionary tracks to warmer effective temperatures at lower metallicities. The observed average spectral subtypes of RSGs in these galaxies shifts from $M 2 I$ in the Milky Way to $M 1 I$ in the LMC and $K 5-K 7 I$ in the SMC. The newly derived physical parameters by Levesque et al. $(2005,2006)$ brought the location of the RSGs into much closer agreement with the predictions of stellar evolutionary theory. There is now excellent agreement between Milky Way supergiants and the evolutionary tracks, accurately reproducing this stage of massive star evolution in the Milky Way. Figures $16-18$ show our more massive tracks from 10 to $20 M_{\odot}$ 


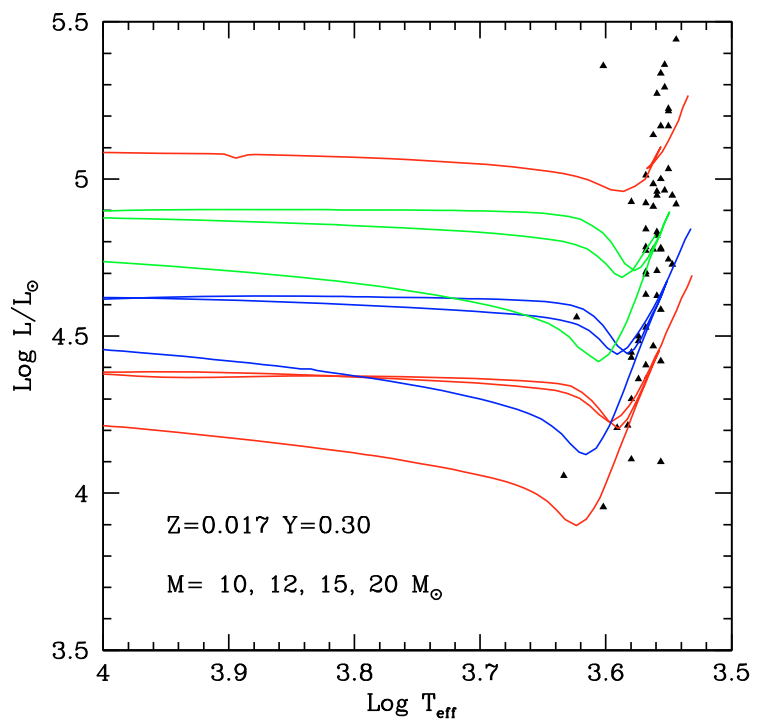

Fig. 16. Evolutionary tracks in the HR diagram, for the composition $Z=0.017, Y=0.30$ for masses $10,12,15$, and $20 M_{\odot}$. Triangles are the Galactic red supergiants from Levesque et al. (2005).

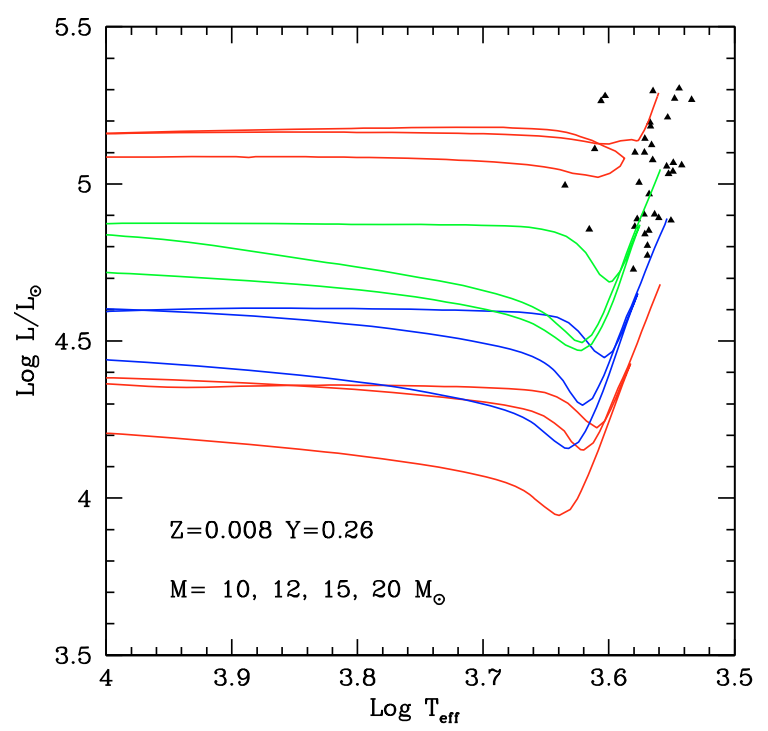

Fig. 17. Evolutionary tracks in the HR diagram, for the composition $Z=0.008, Y=0.26$ for masses $10,12,15$, and $20 M_{\odot}$. Triangles are the LMC red supergiants from Levesque et al. (2006).

displaying also the data from Levesque et al. (2005, 2006) of RSGs in the Milky Way, LMC, and SMC.

\section{Concluding remarks}

Observations of some globular clusters in the Galaxy and the LMC provide evidence of multiple populations and/or significant variations in the helium content, in contrast to traditional scenarios in which globular clusters are simple stellar populations of uniform age and chemical composition. The current database of Padova tracks allows the computation of isochrone sets covering a wide range of ages and enables users to analyse stellar populations (star clusters or galaxies) with different helium enrichment laws, given the extended $Z-Y$ region of the evolutionary models.

The scaled solar database of models and isochrones, presented in Paper I, has been extended in mass to include stellar

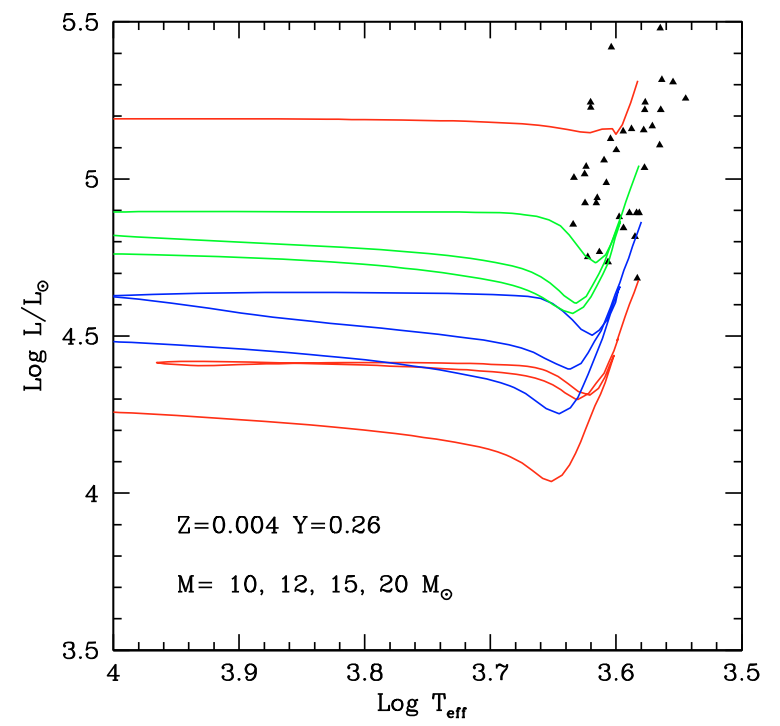

Fig. 18. Evolutionary tracks in the HR diagram, for the composition $Z=0.004, Y=0.26$ for masses $10,12,15$, and $20 M_{\odot}$. Triangles are the SMC red supergiants from Levesque et al. (2006).

evolutionary computations from 2.5 to $20 M_{\odot}$ for the same large region of the $Z-Y$ plane. An important update of this database is the extension of stellar models and isochrones until the end of the TP-AGB phase by means of synthetic models, using the same relations as in Marigo \& Girardi (2007). Revised bolometric corrections by Girardi et al. (2008) are employed to transform Padova isochrones into UBVRIJHK and ACS photometric systems.

In the range of more massive stars presented in this Paper II, our models can be tested in at least two interesting astrophysical problems: 1) Cepheids and the mass-discrepancy problem; 2) massive red supergiants and the problems of their effective temperature (Massey 2003).

Considering the observations of Cepheids in the Galaxy and the Magellanic Clouds (Tamman et al. 2003; Sandage et al. 2004, 2009), we show that the observed instability strip is narrower than the theoretical one, and that the slope of the lower boundary of observed stars coincides with the slope of the faint envelope connecting each of the loops of the theoretical tracks for the chemical composition of the MCs. Because of the small number of Cepheids in open clusters and associations of the Milky Way, we cannot draw the lower boundary of the observed strip as we did for the MCs, but in this case, the loops of the theoretical models can also indicate the location of MW Cepheids in the HR-diagram.

The long-standing open question about the discrepancy between the pulsation and the evolutionary mass of Cepheid stars is considered, by determining the evolutionary mass of Cepheids in MW open clusters (Tamman et al. 2003) from our models (isochrones) and the pulsation mass from the mass dependent PLC relation for fundamental pulsators. The evolutionary mass is found to be higher than the pulsation mass for stars of mass lower than $\sim 6 M_{\odot}$, while for stars more massive than $10 M_{\odot}$ the pulsation mass is higher than the evolutionary one. If we consider extra convective mixing as a plausible solution of the Cepheid mass discrepancy, the parameter that determines the efficiency of the extra mixing should decrease with increasing stellar mass.

Red supergiants in both the Galaxy and the Magellanic Clouds were too cool and luminous compared with evolutionary 
tracks (Massey 2003; Massey \& Olsen 2003) until when Levesque et al. $(2005,2006)$ derived a new effective temperature scale and new bolometric corrections, producing far closer agreement with stellar evolutionary tracks. Our tracks from 10 to $20 M_{\odot}$ for the appropriate chemical composition can reproduce the location in the H-R diagram of red supergiants in the MW and the MCs as given by Levesque et al. (2005, 2006).

A web site has been dedicated to make available the entire database to the scientific community.

In the section YZVAR of the static databases at http:// stev. oapd. inaf. it users can find:

- data files with the information relative to each evolutionary track;

- isochrone files from Paper I with the chemical composition of the computed grids in the $Z-Y$ plane, and age interval in the range $\sim 10.15 \geq \log$ age $/ \mathrm{yr} \geq \sim 9.00$.

In the section YZVAR form of the interactive services a web interface allows users to obtain interpolated isochrones for the entire range of ages, from old (log age/yr $\sim 10.15$ ) to young ages (log age/yr 7.00) and any chemical composition in the provided range of $Y$ and $Z$. The initial and final values of isochrone ages can vary a little more or less, depending on the chemical composition. To compute the isochrones we adopt the interpolation scheme described in Sect. 5.1.

We point out that older isochrones (Paper I) are available in both the static database and the interactive service, while younger isochrones only interactively. All isochrones (old and young) are available through the interactive service for the chosen photometric system (see Sect. 5.3).

In the future, a web interactive interface will provide data of stellar populations in terms of SFR, IMF, chemical composition, and mass loss during the RGB phase.

Acknowledgements. We acknowledge our referee's useful comments and suggestions that helped to improve the presentation of our results. We thank C. Chiosi for his continuous interest and support to stellar evolution computations. We thank A. Weiss and B. Salasnich for help with opacity tables, G. Bono and A. Bressan for useful discussions. We acknowledge financial support from INAF COFIN 2005 "A Theoretical lab for stellar population studies" and from Padova University (Progetto di Ricerca di Ateneo CPDA 052212).

\section{References}

Alexander, D. R., \& Ferguson, J. W. 1994, ApJ, 437, 879

Allard, F., et al. 2000, in From giant planets to cool stars, ed. C. A. Griffith, \& M. S. Marley, ASP Conf. Ser., 212, 127

Alongi, M., Bertelli, G., Bressan, A., \& Chiosi, C. 1991, A\&A, 244, 95

Alongi, M., Bertelli, G., Bressan, A., et al. 1993, A\&AS, 97, 851

Anders, E., \& Grevesse, N. 1989, Geochim. Cosmochim. Acta, 53, 19

Angulo, C., Arnould, M., Rayet, M., et al. 1999, Nucl. Phys. A, 656, 3

Asplund, M., Grevesse, N., Sauval, A. J., et al. 2004, A\&A, 417, 751

Basu, S., \& Antia, H. M. 2008, Phys. Rep., 457, 217

Bemmerer, D., et al. (LUNA Collaboration) 2006, Nucl. Phys. A, 779, 297

Bertelli, G., Bressan, A., Chiosi, C., Fagotto, F., \& Nasi, E. 1994, A\&AS, 106, 275

Bertelli, G., Girardi, L., Marigo, P., \& Nasi, E. 2008, A\&A, 484, 815 (Paper I)

Bessell, M. S. 1990, PASP, 102, 1181

Bessell, M. S., \& Brett, J. M. 1988, PASP, 100, 1134

Böhm-Vitense, E. 1958, Z. Astrophys., 46, 108

Bono, G., Caputo, F., Cassisi, S., et al. 2000, ApJ, 543, 955

Bressan, A., Bertelli, G., \& Chiosi, C. 1981, A\&A, 102, 25

Bressan, A., Fagotto, F., Bertelli, G., \& Chiosi, C. 1993, A\&AS, 100, 647

Caffau, E., Ludwig, H.-G., Steffen, M., et al. 2008, A\&A, 488, 1031

Caputo, F., Castellani, V., Degl'Innocenti, S., Fiorentino, G., \& Marconi, M. 2004, A\&A, 424, 927
Caputo, F., Bono, G., Fiorentino, G., Marconi, M., \& Musella, I. 2005, A\&A, 629,1021

Castellani, V., Chieffi, A., Tornambe, A., \& Pulone, L. 1985, ApJ, 296, 204

Castellani, V., Chieffi, A., \& Straniero, O. 1990, ApJS, 74, 463

Castelli, F., \& Kurucz, R. L. 2003, in Modelling of Stellar Atmospheres, ed. N. E. Piskunov et al. (San Francisco: ASP) IAU Symp., 210, 20

Castor, J. I., Abbott, D. C., \& Klein, R. I. 1975, ApJ, 195, 157

Caughlan, G. R., \& Fowler, W. A. 1988, Atom. Data Nucl. Data Tables, 40, 283

Charbonnel, C., Meynet, G., Maeder, A., et al. 1993, A\&A, 101, 415

Chiosi, C., \& Maeder, A. 1986, ARA\&A, 24, 239

Chiosi, C., Bertelli, G., \& Bressan, A. 1992a, ARA\&A, 30, 305

Chiosi, C., Wood, P. R., Bertelli, G., Bressan, A., \& Mateo, M. 1992b, ApJ, 385, 205

Chiosi, C., Wood, P. R., \& Capitanio, N. 1993, ApJS, 86, 541

Cordier, D., Goupil, M. J., \& Lebreton, Y. 2003, A\&A, 409, 491

de Jager, C., Nieuwenhuijzen, H., \& van der Hucht, K. 1988, A\&AS, 72, 295

de Mink, S. E., Cantiello, M., Langer, N., et al. 2009, A\&A, 497, 243 489, 685

Fagotto, F., Bressan, A., Bertelli, G., \& Chiosi, C. 1994a, A\&AS, 104, 365

Fagotto, F., Bressan, A., Bertelli, G., \& Chiosi, C. 1994b, A\&AS, 105, 29

Ferguson, J. W., Alexander, D. R., Allard, F., et al. 2005, ApJ, 623, 585

Formicola, A., et al. (LUNA Collaboration) 2004, Phys. Lett. B, 591, 61

Fluks, M. A., Plez, B., The, P. S., de Winter, D., et al. 1994, A\&AS, 105, 311

Girardi, L., Bressan, A., Chiosi, C., Bertelli, G., \& Nasi, E. 1996, A\&AS, 117, 113

Girardi, L., Bressan, A., Bertelli, G., \& Chiosi, C. 2000, A\&AS, 141, 371

Girardi, L., Bertelli, G., Bressan, A., et al. 2002, A\&A, 391, 195

Girardi, L., Castelli, F., Bertelli, G., \& Nasi, E. 2007, A\&A, 468, 657

Girardi, L., Dalcanton, J., Williams, B., et al. 2008, PASP, 120, 583

Graboske, H. C., de Witt, H. E., Grossman, A. S., \& Cooper, M. S. 1973, ApJ, 181,457

Grevesse, N. 1991, J. Phys. IV, 1, 181

Grevesse, N., \& Noels, A. 1993, Phys. Scr. T, 47, 133

Grevesse, N., \& Sauval, A. J. 1998, Space Sci. Rev., 85, 161

Grevesse, N., Asplund, M., \& Sauval, A. J. 2007, Space Sci. Rev., 130, 105

Gustafsson, B., Edvardsson, B., Eriksson, K., et al. 2003, in Stellar Atmosphere

Modeling, ed. I. Hubeny et al., ASP Conf. Ser., 288, 331

Guzik, J. A., Watson, L. S., Cox, A. N., et al. 2005, ApJ, 627, 1049

Guzik, J. A., Watson, L. S., \& Cox, A. N. 2006, MSAIt, 77, 389

Haft, M., Raffelt, G., \& Weiss, A. 1994, ApJ, 425, 222

Heger, A., \& Langer, N. 2000, ApJ, 544, 1016

Holtzman, J. A., Burrows, C. J., Casertano, S., et al. 1995, PASP, 107, 1065

Iglesias, C. A., \& Rogers, F. J. 1996, ApJ, 464, 943

Imbriani, G., Costantini, H., Formicola, A., et al. 2004, A\&A, 420, 625

Imbriani, G., et al. (LUNA Collaboration) 2005, EPJ A, 25, 455

Itoh, N., Mitake, S., Iyetomi, H., \& Ichimara, S. 1983, ApJ, 273, 774

Keller, S. C. 2008, ApJ, 677, 483

Kippenhahn, R., Weigert, A., \& Hofmeister, E. 1967, in Methods in

Computational Physics, ed. B. Alder, S. Fernbach, \& M. Rotenberg

(New York: Academic Press), 7, 129

Kudritzki, R. P. 2002, ApJ, 577, 389

Kudritzki, R. P., \& Puls, J. 2000, ARA\&A, 38, 613

Kudritzki, R. P., Gabler, A., Gabler, R., Groth, H. G., \& Pauldrach, A. W. A. 1989, IAU Coll., 113, 67

Landré, V., Prantzos, N., Aguer, P., et al. 1990, A\&A, 240, 85

Lemut, A., et al. (LUNA Collaboration) 2006, Phys. Lett, B, 634, 483

Levesque, E. M., Massey, P., Olsen, K. A. G., et al. 2005, ApJ, 628, 973

Levesque, E. M., Massey, P., Olsen, K. A. G., et al. 2006, ApJ, 645, 1102

Loidl, R., Lancon, A., \& Jorgensen, U. G. 2001, A\&A, 371, 1065

Lucy, L. B., \& Solomon, P. M. 1970, ApJ, 159, 879

Maeder, A. 2009, Physics, Formation and Evolution of Rotating Stars (Berlin, Heidelberg: Springer)

Marigo, P., \& Aringer, B. 2009, A\&A, in press [arXiv: 0907. 3248]

Marigo, P., \& Girardi, L. 2007, A\&A, 469, 239

Marigo, P., Girardi, L., Chiosi, C., \& Wood, P. R. 2001, A\&A, 371, 152

Marigo, P., Girardi, L., Bressan, A., et al. 2008, A\&A, 482, 883

Massey, P. 2003, ARA\&A, 41, 15

Massey, P., \& Olsen, K. A. G. 2003, AJ, 126, 2867

Massey, P., Levesque, E. M., Plez, B., \& Olsen, K. A. G. 2008, in Proc. IAU Symp. 250, 97

Meynet, G., \& Maeder, A. 2000, A\&A, 361, 101

Meynet, G., Maeder, A., Schaller, G., Schaerer, D., \& Charbonnel, C. 1994, A\&AS, 103, 97

Mihalas, D., Hummer, D. G., Mihalas, B. W., \& Däppen, W. 1990, ApJ, 350, 300

Mokiem, M. R., de Koter, A., Vink, J. S., et al. 2007, A\&A, 473, 603

Montalban, J., Miglio, A., Noels, A., Grevesse, N., \& di Mauro, M. P. 2004, ESA SP-559, 574

Neilson, H. R., \& Lester, J. B. 2008, ApJ, 684, 569 
Neilson, H. R., \& Lester, J. B. 2009, ApJ, 690, 1829

Pompéia, L., Hill, V., Spite, M., et al. 2008, A\&A, 480, 379

Pietrinferni, A., Cassisi, S., Salaris, M., \& Castelli, F. 2004, ApJ, 612, 168

Pietrinferni, A., Cassisi, S., Salaris, M., \& Castelli, F. 2006, ApJ, 642, 797

Piotto, G. 2009, in The Ages of Stars, IAU Symp., 258, 233

Piotto, G., Villanova, S., Bedin, L. R., et al. 2005, ApJ, 621, 777

Piotto, G., Bedin, L. R., Anderson, J., et al. 2007, ApJ, 661, L53

Plez, B. 2003, in GAIA Spectroscopy: Science and Technology, ed. U. Munari, ASP Conf. Ser., 298, 189

Rogers, F. J., Swenson, F. J., \& Iglesias, C. A. 1996, ApJ, 456, 902

Romaniello, M., Primas, F., Mottini, M., et al. 2008, A\&A, 488, 731

Salaris, M., Chieffi, A., \& Straniero, O. 1993, ApJ, 414, 580

Salasnich, B., Girardi, L., Weiss, A., \& Chiosi, C. 2000, A\&A, 361, 1023

Salpeter, E. E. 1955, ApJ, 121,161

Sandage, A., Tammann, G. A., \& Reindl, B. 2004, A\&A, 424, 43

Sandage, A., Tammann, G. A., \& Reindl, B. 2009, A\&A, 493, 471

Schaerer, D., Meynet, G., Maeder, A., \& Schaller, G. 1993a, A\&AS, 98, 523

Schaerer, D., Charbonnel, C., Meynet, G., Maeder, A., \& Schaller, G. 1993b, A\&AS, 102, 339
Schaller, G., Schaerer, D., Meynet, G., \& Maeder, A. 1992, A\&AS, 96, 269 Schlattl, H., \& Weiss, A. 1998, in Proc. Neutrino Astrophysics, Ringberg Castle, Germany, Oct. 1997, ed. M. Altmann, W. Hillebrandt et al.

Schroder, K. P., \& Cuntz, M. 2007, A\&A, 465, 593

Scott, P., Asplund, M., Grevesse, N., \& Sauval, A. J. 2009, ApJ, 691, L119

Sirianni, M., Jee, M. J., Benítez, N., et al. 2005, PASP, 117, 1049

Storm, J., Carney, B. W., Gieren, W. P., et al. 2004, A\&A, 415, 531

Tammann, G. A., Sandage, A., \& Reindl, B. 2003, A\&A, 404, 423

Tolstoy, E., Hill, V., \& Tosi, M. 2009, ARA\&A, 47, 371

Vemury, S. K., \& Stothers, R. 1978, ApJ, 225, 939

Villanova, S., Piotto, G., King, I., et al. 2007, ApJ, 663, 296

Vink, J. S., \& de Koter, A. 2005, A\&A, 442, 587

Vink, J. S., de Koter, A., \& Lamers, H. J. G. L. M. 2001, A\&A, 369, 574

Weaver, T. A., \& Woosley, S. E. 1993, Phys. Rep., 227, 65

Weiss, A., \& Schlattl, H. 2000, A\&AS, 144, 487

Weiss, A., Keady, J. J., \& Magee, N. H. Jr. 1990, Atom. Data Nucl. Data Tables, 45, 209

Weiss, A., Serenelli, A., Kitsikis, A., et al. 2005, A\&A, 441, 1129

Yi, S., Demarque, P., Kim, Y.-C., et al. 2001, ApJS, 136, 417 